\title{
HARMONIC VOLUME, SYMMETRIC PRODUCTS, AND THE ABEL-JACOBI MAP
}

\author{
WILLIAM M. FAUCETTE
}

\begin{abstract}
The author generalizes B. Harris' definition of harmonic volume to the algebraic cycle $W_{k}-W_{k}^{-}$for $k>1$ in the Jacobian of a nonsingular algebraic curve $X$. We define harmonic volume, determine its domain, and show that it is related to the image $\nu$ of $W_{k}-W_{k}^{-}$in the Griffiths intermediate Jacobian. We derive a formula expressing harmonic volume as a sum of integrals over a nested sequence of submanifolds of the $k$-fold symmetric product of $X$. We show that $\nu$, when applied to a certain class of forms, takes values in a discrete subgroup of $\mathbf{R} / \mathbf{Z}$ and hence, when suitably extended to complexvalued forms, is identically zero modulo periods on primitive forms if $k \geq 2$. This implies that the image of $W_{k}-W_{k}^{-}$is identically zero in the Griffiths intermediate Jacobian if $k \geq 2$. We introduce a new type of intermediate Jacobian which, like the Griffiths intermediate Jacobian, varies holomorphically with moduli, and we consider a holomorphic torus bundle on Torelli space with this fiber. We use the relationship mentioned above between $\nu$ and harmonic volume to compute the variation of $\nu$ when considered as a section of this bundle. This variational formula allows us to show that the image of $W_{k}-W_{k}^{-}$ in this intermediate Jacobian is nondegenerate.
\end{abstract}

\section{INTRODUCTION}

Let $X$ be a Riemann surface and let $J=J(X)$ be its Jacobian variety. The Abel-Jacobi map $I_{1}: X \rightarrow J$ extends naturally to the $k$ th symmetric product $X_{k}$ giving a map $I_{k}: X_{k} \rightarrow J$. In this way $X_{k}$ defines an algebraic $k$-cycle on $J$, which is universally denoted $W_{k}$ in the literature. The algebraic $k$-cycle $W_{k}$ may be viewed as the image under the Abel-Jacobi map of effective divisors of degree $k$ on $X$. Let $W_{k}^{-}$be the image of $W_{k}$ under the group involution on $J(X)$. We remark that the algebraic cycle $W_{k}-W_{k}^{-}$is homologous to zero.

In the simplest case, that of two points $p$ and $q$ on a Riemann surface $X$, being homologous is equivalent to being algebraically equivalent as algebraic 0 -cycles. In fact, Lefschetz showed that homological equivalence and algebraic equivalence coincide for divisors, that is, algebraic cycles of codimension one, although it is now known that this is the exception rather than the rule in higher codimensions. In 1969, with the foundational work of P. Griffiths, it was shown that homological equivalence is, in general, strictly weaker than algebraic

Received by the editors June 23, 1988 and, in revised form, October 16, 1990.

1980 Mathematics Subject Classification (1985 Revision). Primary 14C10; Secondary 14H15, $14 \mathrm{H} 40,14 \mathrm{~K} 30$.

Key words and phrases. Harmonic volume, algebraic equivalence. 
equivalence [G3, G4]. This result spawned a good deal of work to determine the exact relationship between algebraic equivalence and homological equivalence.

For instance, the algebraic $k$-cycle $W_{k}$ determines an element $w_{g-k}$ in the algebraic equivalence ring of $J(X)$. It is known that Poincaré's formulas

$$
i ! w_{i}=w_{1}^{i}
$$

hold in the cohomology ring $H^{*}(J ; \mathbf{Z})$. A. Collino showed in 1975 that these formulas also hold in the algebraic equivalence ring of a hyperelliptic Riemann surface $X[\mathrm{Co}]$.

In 1983 G. Ceresa [C] succeeded in showing that for a generic Riemann surface $X, W_{k}$ and $W_{k}^{-}$are algebraically inequivalent if $1 \leq k \leq g-2$. Of course, $W_{g-1}$ and $W_{g-1}^{-}$are always algebraically equivalent since they are homologous divisors in $J$. Ceresa succeeded in proving his result by assuming that $W_{1}$ and $W_{1}^{-}$are algebraically equivalent for curves $X$ lying in a Zariski open set in moduli space and then using differential calculus in moduli space to derive certain relations about the vanishing of linear functionals on $H^{3}(J(X) ; \mathbf{C})$ for all curves $X$. He then produces a reducible curve for which these relations fail to hold, thus reaching a contradiction. The general result is then obtained by induction.

A similar type of result was obtained by Ceresa and Collino [CC] in 1983 when they showed that, for a generic 3-fold $F$ in $\mathbf{P}^{4}$ with one ordinary double point, the difference of the two generators in the smooth quadric $H$, which is the inverse image in the proper transform of $F$ of the singular point under the blowing up of $\mathbf{P}^{4}$ at the singular point, is not algebraically equivalent to zero, even though Griffiths has shown that it is homologous to zero.

The difficulty with this approach is that although Ceresa's theorem establishes that $W_{1}$ and $W_{1}^{-}$are algebraically inequivalent for a generic curve $X$, the theorem provides no way of determining, for a given Riemann surface $X$, whether $W_{1}$ and $W_{1}^{-}$are algebraically equivalent.

In his 1983 paper in Acta Mathematica [BH1], B. Harris contributed to the ability to determine when $W_{1}$ and $W_{1}^{-}$are algebraically inequivalent by introducing the technique of harmonic volume. Let $X$ be a nonsingular algebraic curve of genus $g \geq 3$ and let $\theta_{1}, \theta_{2}, \theta_{3}$ be three real harmonic 1-forms on $X$ with integer periods. Choose a basepoint $p \in X$ and define a map $J_{1}: X \rightarrow T^{3}$ by

$$
J_{1}(x)=\left(\int_{p}^{x} \theta_{1}, \int_{p}^{x} \theta_{2}, \int_{p}^{x} \theta_{3}\right) \text { modulo } \mathbf{Z}^{3} .
$$

By imposing the conditions that $\int_{X} \theta_{i} \wedge \theta_{j}=0$ for $1 \leq i<j \leq 3$, we are assured that the image $J_{1} X$ in $T^{3}$, as a singular 2-cycle, is the boundary of some 3-chain $C_{3}$, which is unique modulo 3-cycles. We define the harmonic volume of $\theta_{1}, \theta_{2}, \theta_{3}$ to be the integral

$$
\int_{C_{3}} d x_{1} \wedge d x_{2} \wedge d x_{3}
$$

where $J_{1}^{*}\left(d x_{i}\right)=\theta_{i}$.

The importance of the concept of harmonic volume lies in the following two facts. First, the value of the linear functional $\nu$ which is the image of the algebraic cycle $W_{1}-W_{1}^{-}$in the Griffiths intermediate Jacobian associated 
to the Jacobian of $X$ is twice the value of harmonic volume. Second, in a concrete computational sense, an equivalent definition of harmonic volume is as the iterated integral

$$
\int_{\gamma}\left(h_{1} \theta_{2}-\eta_{12}\right) \text { modulo } \mathbf{Z}
$$

where $\gamma$ is a path on $X$ Poincare dual to the cohomology class of $\theta_{3}, h_{1}$ is a function on $\gamma$ obtained by integrating $\theta_{1}$, and $\eta_{12}$ is a 1 -form on $X$ satisfying $d \eta_{12}=\theta_{1} \wedge \theta_{2}$. It is this interpretation of harmonic volume as an iterated integral which allows its explicit computation.

If $W_{1}$ is algebraically equivalent to $W_{1}^{-}$, then $\nu$, suitably extended to a C-linear functional $\tilde{\nu}$ on complex-valued forms, must vanish on $(3,0)$-forms, since $W_{1}$ and $W_{1}^{-}$are both contained in an algebraic surface. Thus, in order for $W_{1}$ and $W_{1}^{-}$to be algebraically equivalent, it is necessary that twice the harmonic volume vanish on every 3-tuple of holomorphic differentials. This result was known to Hodge and we recall it here in its higher dimensional analog:

Proposition 1.1. If $W_{k}$ is algebraically equivalent to $W_{k}^{-}$in $J$, then the C-linear map $\tilde{\nu}$, considered as a linear functional on $H^{2 k+1,0}(J) \oplus \cdots \oplus H^{k+1, k}(J)$, is zero modulo periods except possibly on $H^{k+1, k}(J)$.

Using the technique of harmonic volume, Harris shows that the Fermat curve of genus three is a specific example of a curve $X$ whose image $W_{1}$ in its Jacobian is not algebraically equivalent to the image of $W_{1}$ under the group involution [BH2].

We remark that Harris' harmonic volume necessarily vanishes if $X$ is a hyperelliptic Riemann surface since the image of a hyperelliptic Riemann surface in its Jacobian is merely translated under the group involution. It is conjectured that the converse also holds: If the harmonic volume map is identically zero on a Riemann surface $X$, then $X$ is hyperelliptic. A result by M. Pulte [P] in this direction shows that if the harmonic volume vanishes there must exist on $X$ a distinguished $(g-1)$ th root of the canonical divisor.

The goal of this paper is to generalize Harris' definition of harmonic volume to the algebraic cycles $W_{k}-W_{k}^{-}$for $k>1$. This will be accomplished by generalizing the idea of choosing $p$ real harmonic 1-forms with integral periods and integrating from a fixed basepoint to give a map $J_{l}: X_{l} \rightarrow T^{p}$, for $1 \leq l \leq\left[\frac{p}{2}\right]$, of the $l$ th symmetric product of $X$ into a $p$-dimensional torus. We first ask what condition must be imposed on the choice of these 1-forms in order to insure that the image will be the boundary of some chain. The answer to this question will be shown to depend only on the cohomology class of the wedge product of these forms when considered as representing elements of $H^{1}(J(X) ; \mathbf{R})$. We will call a class in $H^{p}(J(X) ; \mathbf{Z})$ l-good if it is represented by a wedge product of $p$ real harmonic 1 -forms with integral periods such that the image of $X_{l}$ under the associated map $J_{l}: X_{l} \rightarrow T^{p}$ is the boundary of some $(2 l+1)$-chain.

Our first result is best expressed in the language of filtrations. The Lefschetz decomposition of any $p$-form on $J(X)$

$$
\sum_{r=0}^{[p / 2]} L^{r}\left(\theta^{(r)}\right)
$$


where $L$ is the Lefschetz operator and $\theta^{(r)}$ is a primitive $(p-2 r)$-form, provides a filtration of the real cohomology of $J(X)$ :

$$
\begin{aligned}
L^{0}\left(\theta^{(0)}\right) & \subset L^{0}\left(\theta^{(0)}\right)+L^{1}\left(\theta^{(1)}\right) \subset \cdots \subset \sum_{r=0}^{[p / 2]-2} L^{r}\left(\theta^{(r)}\right) \\
& \subset \sum_{r=0}^{[p / 2]-1} L^{r}\left(\theta^{(r)}\right) \subset \sum_{r=0}^{[p / 2]} L^{r}\left(\theta^{(r)}\right)=H^{p}(J(X), \mathbf{R}) .
\end{aligned}
$$

Let $G^{l}$ be the subgroup of $H^{p}(J(X) ; \mathbf{Z})$ generated by $l$-good $p$-forms and define $G_{\mathbf{R}}^{l}$ to be the subspace $G^{l} \otimes \mathbf{R}$ of $H^{2 k+1}(J ; \mathbf{R})$ and $G_{\mathbf{C}}^{l}$ to be the subspace $G^{l} \otimes \mathbf{C}$ of $H^{2 k+1}(J ; \mathbf{C})$. Then the groups $G_{\mathbf{R}}^{l}$ also provide such a filtration:

$$
G_{\mathbf{R}}^{1} \subset G_{\mathbf{R}}^{2} \subset \cdots \subset G_{\mathbf{R}}^{[p / 2]-1} \subset G_{\mathbf{R}}^{[p / 2]} \subset H^{p}(J ; \mathbf{R}) .
$$

Our first result is that these two filtrations agree. In particular, a decomposable integral $(2 k+1)$-form is $k$-good if and only if the last term of its Lefschetz decomposition vanishes.

B. Harris has shown that in the case $k=1$, the class of good forms coincides with the class of primitive forms. The result just cited shows that for $k \geq 2$, the class of good forms is strictly larger than the class of primitive forms.

In $\S 3$ we limit our attention to $p=2 k+1, l=k$. Here we will use the term good rather than $k$-good. By choosing a chain in $T^{2 k+1}$ with boundary equal to the image $J_{k} X_{k}$ we define harmonic volume to be the linear functional on $(2 k+1)$-forms obtained by integrating modulo $\mathbf{Z}$ over this chain. Just as in Harris' paper, the homomorphism $\nu$ on good forms is twice the value of harmonic volume. We go on to show that values of harmonic volume on good forms lying in $G^{k-1}$ lie in a discrete subgroup of $\mathbf{R} / \mathbf{Z}$. This result will be used in $\S 4$ to show that the linear functional $\nu$ vanishes identically on $G^{k-1}$. In particular, $\nu$ vanishes on all primitive forms if $k \geq 2$.

This last result generalizes a remark of G. Ceresa [C, Remark 3.15]. The content of Ceresa's remark is that the map $\nu$, suitably extended to a complexlinear map $\tilde{\nu}$, when restricted to primitive $(g, 0)$ forms, gives a (multivalued) section (depending on the choice of chain with boundary $W_{k}-W_{k}^{-}$) of the dual of the Hodge bundle on moduli space which vanishes modulo periods along the divisor of curves $X$ containing a pencil of divisors of degree $k+1$. The result cited at the end of the last paragraph shows that this section vanishes identically if $k \geq 2$. The vanishing of this section for $k \geq 2$ may be given a proof independent of harmonic volume, and we do so here.

Proposition 1.2. Let $X$ be a nonsingular algebraic curve of genus $g \geq 2 k+1$. Let $\theta=\theta_{1} \wedge \cdots \wedge \theta_{2 k+1}$ be a decomposable $(r, 2 k+1-r)$-form on $J$.

If $r<\left[\frac{k}{2}\right]$ or if $r>2 k+1-\left[\frac{k}{2}\right]$, then $\tilde{\nu}(\theta)=0$ modulo periods. In particular, if $k \geq 2, \tilde{\nu}$ is identically zero modulo periods on $(2 k+1,0)$ forms.

Proof. We may suppose $k \geq 2$. Let $l=\left[\frac{k}{2}\right]$ and define

$$
\widetilde{D}_{2 k+1}=m_{\#}\left(W_{l} \times D_{2(k-l)+1}+D_{2 l+1} \times W_{k-l}^{-}\right),
$$

where $\partial D_{2 j+1}=W_{j}-W_{j}^{-}$. A computation shows that $\widetilde{D}_{2 k+1}$ is homologous to $\left(\begin{array}{l}k \\ l\end{array}\right)$ times $D_{2 k+1}$, since $W_{l} \times W_{k-l}$ is a $\left(\begin{array}{l}k \\ l\end{array}\right)$-sheeted cover of $W_{k}$. Computing 
the integral of $\theta$ over $\widetilde{D}_{2 k+1}$ by pulling back the integrand to $W_{l} \times D_{2(k-l)+1}+$ $D_{2 l+1} \times W_{k-l}^{-}$yields an expression of the integral as a linear combination of products of integrals over $W_{l}$ and $D_{2(k-l)+1}$ and of integrals over $W_{k-l}$ and $D_{2 l+1}$. The hypotheses force all the integrals over $W_{k-l}$ and $W_{l}$ to be zero, and it follows that $\tilde{\nu}(\theta)$ is a $\left(\begin{array}{l}k \\ l\end{array}\right)$ torsion point. A continuity argument similar to the one in the proof of Proposition 4.1 as $X$ varies over Torelli space then shows that $\tilde{\nu}(\theta)$ is zero.

We note that in the example given in [BH2] using the Fermat curve of degree 4 , it is shown that $\tilde{\nu}$ does not vanish identically on $H^{3,0}(X)$ if the genus of $X$ is 3, whereas by Ceresa's remark, if $X$ is hyperelliptic of genus $3, \tilde{\nu}$ is identically zero. This is easily seen since the image of a hyperelliptic $X$ in its Jacobian is translated under the group involution.

In $\S 4$ we use a continuity argument as $X$ varies in Torelli space together with the result that values of harmonic volume on good forms in $G^{k-1}$ lie in a discrete subgroup of $\mathbf{R} / \mathbf{Z}$ to show that $\nu$ is identically zero on $G^{k-1}$. In particular, this shows that for $k \geq 2$, the image of $W_{k}-W_{k}^{-}$in the intermediate Jacobian $\mathscr{J}(J(X))$ of the Jacobian of $X$ is identically zero, and hence the Griffiths primitive intermediate Jacobian cannot be used to determine the algebraic equivalence of $W_{k}$ and $W_{k}^{-}$.

The Hodge filtration on $H^{2 k+1}(J)$, induces a filtration on the subspace $G_{\mathbf{C}}^{k}$ of $H^{2 k+1}(J)$ generated by good forms. This filtration is given by $F^{d} G_{\mathbf{C}}^{k}=$ $G^{2 k+1,0}(J)+\cdots+G^{d, 2 k+1-d}(J)$. Since $\overline{F^{k+1} G_{\mathbf{C}}^{k}} \cap G_{\mathbf{R}}^{k}=0$, the natural R-linear map

$$
G_{\mathbf{R}}^{k} \rightarrow G_{\mathbf{C}}^{k} / \overline{F^{k+1} G_{\mathbf{C}}^{k}} \cong F^{k+1} G_{\mathbf{C}}^{k},
$$

is an isomorphism. This gives $G_{\mathbf{R}}^{k}$ a natural complex structure that, by the work of Griffiths, varies holomorphically with the complex structure on $J$.

We consider the homomorphism $\nu$ as a section of a holomorphic torus bundle defined as follows. The linear functional $\nu$ is an element of $\operatorname{Hom}\left(G^{k}, \mathbf{R} / \mathbf{Z}\right)$ $\cong \operatorname{Hom}_{\mathbf{C}}\left(G_{\mathbf{R}}^{k}, \mathbf{C}\right) / \operatorname{Hom}\left(G^{k}, \mathbf{Z}\right)$. Using the complex structure on $G_{\mathbf{R}}^{k}$ constructed above, this quotient space is a compact complex Lie group similar to Griffiths' intermediate Jacobian [G2]. In fact, it is merely $\left(F^{k+1} G_{\mathbf{C}}^{k}\right)^{*}$ modulo the lattice $G^{*}$. On Torelli space we construct a bundle with fiber $\left(F^{k+1} G_{\mathbf{C}}^{k}\right)^{*} / G^{*}$. The homomorphism $\nu$ then provides a holomorphic section of this bundle, a result which is also essentially due to Griffiths. Using harmonic volume as a computational tool, we then derive a formula computing the "vertical codifferential" of $\nu$, a concept made precise in $\S 5$.

In $\S 5$ we show that harmonic volume is nondegenerate by using the formula for the codifferential mentioned above to compute the differential of the harmonic volume map at a point of Torelli space representing a hyperelliptic Riemann surface. We note that the differential at this point is injective on the normal space to the $(2 g-1)$-dimensional hyperelliptic locus. This result suggests that the use of an intermediate Jacobian based on periods of good forms rather than on periods of primitive forms might be fruitful in achieving a version of Abel's theorem in higher dimensions for classifying algebraic cycles up to algebraic equivalence.

The research appearing here is taken from my Ph.D. dissertation, done at Brown University under the direction of Bruno Harris. 
I wish to thank Joe Harris, Bill Fulton, Jean-Luc Brylinski, Bob MacPherson, John Hughes, David Zelinsky, Alan Landman, and Bob Accola for their professional insights and assistance at various stages in my research. Special thanks go to Alan Landman whose diligence in reading my dissertation helped prevent critical errors from going into print. Further, I would like to thank the geometry/topology faculty of The University of Georgia, particularly Ted Shifrin, Robert Varley, and Roy Smith, for their help. My sincerest gratitude also goes to Roy Smith and Giuseppe Ceresa for helpful correspondences.

My highest thanks and gratitude go to Bruno Harris, whose research inspired this work.

\section{GOOD FORMS}

In the one-dimensional case, B. Harris defines a map $J_{1}: X \rightarrow T^{3}$ by integrating modulo $\mathbf{Z}$ three real harmonic 1 -forms with integral periods along a path. In order to determine the domain of harmonic volume, he determines what constraints must be imposed on the 1 -forms in order to ensure that the image $J_{1} X$ in $T^{3}$ is the boundary of some 3-chain. As remarked in the introduction, the condition that the image $J_{1} X$ in $T^{3}$ is a boundary depends only on the wedge product of the three 1-forms, considered as forms on $J(X)$. He discovers that the constraints can be expressed in terms of the vanishing of the natural skewsymmetric pairing of 1 -forms on $X$ defined by $\theta_{i} \cdot \theta_{j}=\int_{X} \theta_{i} \wedge \theta_{j}$. Harris then shows that the class of decomposable integral 3-forms on $J(X)$ which satisfy these constraints is precisely the set of primitive decomposable integral 3-forms.

In this section we will determine the domain of our higher dimensional harmonic volume by choosing real integral harmonic 1 -forms $\theta_{1}, \ldots, \theta_{p}$ on $X$ and defining a map $J_{l}: X_{l} \rightarrow T^{p}$ for each $l, 1 \leq l \leq\left[\frac{p-1}{2}\right]$. We then ask what condition must be imposed on the wedge product $\theta_{1} \wedge \cdots \wedge \theta_{p}$, considered as representing an element of $H^{p}(J(X) ; \mathbf{Z})$, in order to ensure that the image $J_{l} X_{l}$ is the boundary of some chain in $T^{p}$. We then proceed naively using the fact that $J_{l} X_{l}$ is a boundary if and only if it represents the zero element in $H^{p}\left(T^{p} ; \mathbf{Q}\right)^{*}$.

Let $X$ be a Riemann surface of genus $g \geq p \geq 3$ for a fixed natural number $p$. Let $J=J(X)$ be its Jacobian variety. Let $\theta_{1}, \ldots, \theta_{p}$ be real harmonic 1 -forms on $J(X)$ with integer periods. For each $l, 1 \leq l \leq\left[\frac{p-1}{2}\right]$, define $J_{l}: X_{l} \rightarrow T^{p}$ by

$$
J_{l}\left(x_{1}, \ldots, x_{l}\right)=\left(\sum_{i=1}^{l} \int_{p}^{x_{i}} \theta_{1}, \ldots, \sum_{i=1}^{l} \int_{p}^{x_{i}} \theta_{p}\right) \text { modulo } \mathbf{Z}^{p} .
$$

We define $\theta_{1} \wedge \cdots \wedge \theta_{p}$ to be an $l$-good $p$-form if $J_{l} X_{l}$ is the boundary of some $(2 l+1)$-chain in $T^{p}$. By viewing $J_{l}(x)$ as a linear functional on the subspace $V$ of $H^{1}(X ; \mathbf{Q})$ generated by $\theta_{1}, \ldots, \theta_{p}$, it is immediate that the definition of good depends only on $V$ and not on the individual 1-forms. Hence, the definition of $l$-good depends only on the wedge product of these 1 -forms.

We now wish to determine which $p$-forms are $l$-good.

Lemma 2.1. $J_{l} X_{l}$, as a singular $2 l$-cycle, is the boundary of some $(2 l+1)$-chain in $T^{p}$ if and only if 


$$
\sum_{\tau \in S_{2 l}}(\operatorname{sgn} \tau) \prod_{m=1}^{l}\left(\theta_{\tau \sigma(2 m-1)} \cdot \theta_{\tau \sigma(2 m)}\right)=0
$$

for all order preserving injections $\sigma:\{1, \ldots, 2 l\} \rightarrow\{1, \ldots, p\}$.

Proof. $J_{l} X_{l}$ is a boundary in $T^{p}$ if and only if $\int_{J_{l} X_{l}} \psi=0$ for all closed $2 l$-forms $\psi$ on $T^{p}$. Since $H^{2 l}\left(T^{p}\right)$ equals $\Lambda^{2 l} H^{1}\left(T^{p}\right)$, it is necessary and sufficient to show that $\int_{J_{I} X_{l}} \psi=0$ for all $\psi$ 's of the form $d x_{\sigma(1)} \wedge \cdots \wedge d x_{\sigma(2 l)}$, where $\sigma:\{1, \ldots, 2 l\} \rightarrow\{1, \ldots, p\}$ is an order preserving injection. Pulling this integral back to $X_{l}$ and using the fact that $H^{1}\left(X_{l} ; Z\right)$ is isomorphic to the subring of $H^{1}\left(X^{l} ; \mathbf{Z}\right)$ of elements invariant under the natural action of the symmetric group $S_{l}$ (see [M]), this integral may be explicitly computed as a product of integrals over $X$. This computation yields the result.

Let $B_{l, \sigma}=\sum_{\tau \in S_{2 l}}(\operatorname{sgn} \tau) \prod_{m=1}^{l}\left(\theta_{\tau \sigma(2 m-1)} \cdot \theta_{\tau \sigma(2 m)}\right)$.

We notice that the condition in Lemma 2.1 contains products of terms of the form $\theta_{i} \cdot \theta_{j}$. Such terms may be obtained by taking the interior product of $\theta_{1} \wedge \cdots \wedge \theta_{2 k+1}$ with powers of the fundamental class $\eta$ of the image of $X$ in $J$, if we consider these forms simply as elements of dual exterior algebras. We remark that if $A_{i}, B_{i}$ for $1 \leq i \leq g$ is a canonical basis for $H^{1}(X ; \mathbf{Z})$, then the fundamental 2-form $\Omega$ on $J$ is equal to $\sum_{i=1}^{g} B_{i} \wedge A_{i}$. Further, if $a_{i}, b_{i}$ for $1 \leq i \leq g$ is the dual basis for $H_{1}(X ; \mathbf{Z}) \cong H_{1}(J ; \mathbf{Z})$, then $\eta=\sum_{i=1}^{g} b_{i} \wedge a_{i}$ is the homology class of the image of $X$ in $J$. Hence, we see how taking the interior product with powers of $\eta$ and the exterior product with powers of $\Omega$, that is, powers of the Lefschetz operator, might be related. This motivates the following linear algebra construction.

Using the fact that the cohomology ring $H^{*}(J ; \mathbf{Q})$ is the exterior algebra on $H^{1}(J ; \mathbf{Q})$ and that $H_{*}(J ; \mathbf{Q})$ is the dual exterior algebra, consider the following linear algebra construction. For $n \leq m$, we define the pseudocap product,

$$
\widetilde{ก}: H^{m}(J ; \mathbf{Q}) \otimes H_{n}(J ; \mathbf{Q}) \rightarrow H^{m-n}(J ; \mathbf{Q})
$$

by setting $\theta_{1} \wedge \cdots \wedge \theta_{m} \tilde{\cap} c_{1} \wedge \cdots \wedge c_{n}$ equal to

$$
K(m, n) \sum_{\sigma \in S_{m}}(\operatorname{sgn} \sigma) \theta_{\sigma(1)}\left(c_{1}\right) \cdots \theta_{\sigma(n)}\left(c_{n}\right) \theta_{\sigma(n+1)} \wedge \cdots \wedge \theta_{\sigma(m)},
$$

where

$$
K(m, n)=\frac{1}{(m-n) !}(-1)^{n(m-n)+\frac{n(n-1)}{2}} .
$$

An analogous construction for $n \geq m$ defines the ordinary cap product.

Now define

$$
P_{l}: H^{p}(J ; \mathbf{Q}) \rightarrow H^{p-2 l}(J ; \mathbf{Q})
$$

to be the map taking $\theta$ to $\theta \widetilde{\cap} \eta^{l}$. This is simply the interior product described above between the dual exterior algebras.

Lemma 2.2. Let $\theta=\theta_{1} \wedge \cdots \wedge \theta_{p}$ be a decomposable rational $p$-form. Then

$$
P_{l}(\theta)=\frac{1}{2^{l}(p-2 l) !} \sum_{\mu \in S_{p}}(\operatorname{sgn} \mu) \theta_{\mu(2 l+1)} \wedge \cdots \wedge \theta_{\mu(p)} \prod_{m=1}^{l}\left(\theta_{\mu(2 m-1)} \cdot \theta_{\mu(2 m)}\right) .
$$


Proof. This lemma follows by a straightforward computation by induction on $l$ and the fact that $\theta \widetilde{\cap} \eta^{l}=\left(\theta \widetilde{\cap} \eta^{l-1}\right) \widetilde{\cap} \eta$ for all natural numbers $l \geq 2$.

Hence, pseudocap product with $\eta^{l}$ has the effect of sequentially removing $l$ pairs of 1-forms, taking their product under the skew-symmetric bilinear form, and then taking the product of these $l$ numbers as the coefficient for the wedge product of the remaining $(p-2 l) 1$-forms, at least up to a nonzero multiplicative constant. It should be evident to the reader how this is related to the condition in Lemma 2.1.

Proposition 2.3. Let $J_{l}$ be defined by the linearly independent integral harmonic forms $\theta_{1}, \ldots, \theta_{p} . J_{l} X_{l}$ is the boundary of some $(2 l+1)$-chain in $T^{p}$ if and only if

$$
P_{l}\left(\theta_{1} \wedge \cdots \wedge \theta_{p}\right)=0 .
$$

Proof. Using the result of Lemma 2.2 and a combinatorial argument, it can be shown that the coefficients of the linearly independent terms in $P_{l}(\theta)$ are precisely the numbers $B_{l, \sigma}$ from Lemma 2.1. Hence, $P_{l}(\theta)=0$ if and only if all the $B_{l, \sigma}$ 's are zero. Now Lemma 2.1 completes the proof.

Now we may begin studying the kernel of $P_{l}$.

Proposition 2.4. Let $\theta=\theta_{1} \wedge \cdots \wedge \theta_{p}$ be a decomposable p-form in $H^{p}(J ; \mathbf{Q}) \cong$ $\Lambda^{p} H^{1}(J ; \mathbf{Q})$. Then there exist nonnegative integers $s$ and $t$ with $2 s+t=p$ and real harmonic 1-forms $A_{i}, B_{j}$ for $1 \leq i \leq s+t, 1 \leq j \leq s$, with $A_{i} \cdot B_{j}=\delta_{i j}$ and $A_{i} \cdot A_{j}=0=B_{i} \cdot B_{j}$ for all $i$ and $j$, and

$$
\theta=c A_{1} \wedge B_{1} \wedge \cdots \wedge A_{s} \wedge B_{s} \wedge A_{s+1} \wedge \cdots \wedge A_{s+t},
$$

for some nonzero rational constant $c$. Further, the numbers $s$ and $t$ are unique. Proof. By considering $\theta$ as representing a $p$-plane in $H^{1}(J ; \mathbf{Q})$ and the pairing $(\alpha, \beta) \mapsto \int_{X} \alpha \wedge \beta$ as a skew-symmetric bilinear form, this proposition reduces to an elementary linear algebra fact about canonical representations of skewsymmetric bilinear forms over a field.

Define a decomposable rational $p$-form $\theta$ to be of wedge type $(s, t)$ if $s$ and $t$ are as in Proposition 2.4 and write $\theta=\theta^{s, t}$.

Let $G^{l} \subset H^{p}(J ; \mathbf{Z})$ be the subgroup generated by all $l$-good $p$-forms and let $G_{\mathbf{Q}}^{l}$ be the vector space $G^{l} \otimes_{\mathbf{Z}} \mathbf{Q}$. Let $O_{l} \subset H^{p}(J ; \mathbf{Z})$ be the subgroup generated by all decomposable integral $p$-forms where, possibly after some rearrangement of the $\theta_{i}$ 's, one has $\theta_{i} \cdot \theta_{j}=0$ for $1 \leq i, j \leq p-l+1$. The class $O_{l}$ will be called the set of l-orthogonal $p$-forms. The Lefschetz operator is defined to be the map

$$
\begin{aligned}
L: H^{p}(J ; \mathbf{Q}) & \rightarrow H^{p+2}(J ; \mathbf{Q}) \\
\theta & \mapsto \Omega \wedge \theta .
\end{aligned}
$$

Proposition 2.5. Let $\theta^{s, t}$ be a decomposable rational $p$-form of wedge type $(s, t)$ representing an element in $H^{p}(J ; \mathbf{Q})$.

(a) $\theta^{s, t} \widetilde{\cap} \eta^{l}=0$ if and only if $l>s$.

Consequently, $\theta^{s, t}$ is a generator of $G_{\mathbf{Q}}^{l}$ if and only if $l>s$.

(b) $\theta^{s, t}$ is a generator of $O^{l} \otimes_{\mathbf{Z}} \mathbf{Q}$ if and only if $l>s$.

(c) $L^{g-p+l}\left(\theta^{s, t}\right)=0$ if and only if $l>s$. 
Hence, for decomposable rational p-forms $\theta$, one has

$\theta \in G_{\mathbf{Q}}^{l}$ if and only if $\theta \in O_{l} \otimes_{\mathbf{Z}} \mathbf{Q}$ if and only if $\theta \in \operatorname{ker} L^{g-p+l}$.

Proof. Using Proposition 2.4, write $\theta$ in the canonical form given there, and complete the set of 1 -forms to a canonical basis $A_{i}, B_{i}, 1 \leq i \leq g$, for $H^{1}(J ; \mathbf{Q})$. Then the fundamental 2-form on $J$ is given by $\Omega=\sum_{i=1}^{g} B_{i} \wedge$ $A_{i}$. From the paragraph after the proof of Lemma 2.2 which describes the pseudocap product, one concludes that $\theta^{s, t} \widetilde{\cap} \eta^{s}$ is a rational constant times $A_{s+1} \wedge \cdots \wedge A_{s+t}$, and $\theta^{s, t} \widetilde{\cap} \eta^{l}$ is zero if $l>s$ since all the $A_{i}$ 's are orthogonal.

A generator of $O_{l} \otimes_{\mathrm{Z}} \mathbf{Q}$ must represent a $p$-plane in $H^{1}(J ; \mathbf{Q})$ which contains a $(p-l+1)$-dimensional totally isotropic subspace with respect to the skewsymmetric pairing of 1 -forms. This is possible if and only if $s+t \geq p-l+1$, which is equivalent to $l>s$.

Likewise, $L^{q}(\theta)=0$ if and and only if $q+s+t>g$, so $L^{g-p+l}(\theta)=0$ if and only if $g-p+l+s+t>g$, which is equivalent to $l>s$.

Proposition 2.6. Let $\theta$ be a p-form on $J$ representing an element of $H^{p}(J ; \mathbf{Q})$. Then for $1 \leq l \leq\left[\frac{p-1}{2}\right], \theta$ is in $\operatorname{ker} L^{g-p+l}$ if and only if $\theta$ is in $G_{\mathbf{Q}}^{l}$.

Proof. By Proposition 2.5 and the linearity of the Lefschetz operator, it is clear that $G_{\mathbf{Q}}^{l} \subset \operatorname{ker} L^{g-p+l}$.

For the reverse inclusion, choose a canonical basis $A_{i}, B_{i}, 1 \leq i \leq g$, for $H^{1}(J ; \mathbf{Z})$. Write

$$
\theta=\sum_{J \in J} \theta_{J} \wedge C_{j_{1}} \wedge \cdots \wedge C_{j_{t}}
$$

where $C_{i}$ equals either $A_{i}$ or $B_{i}, \theta_{J}$ consists of a sum of forms containing only wedge products of $s$ pairs $A_{i} \wedge B_{i}$, and the sum is over all distinct singleton endings $C_{j_{1}} \wedge \cdots \wedge C_{j_{t}}$. Since forms with different singleton endings are linearly independent, $L^{q}(\theta)=0$ if and only if $L^{q}\left(\theta_{J}\right) \wedge C_{j_{1}} \wedge \cdots \wedge C_{j_{t}}=0$ for all singleton endings. However, a simple computation shows that $L^{g-p+l}\left(\theta_{J}\right) \wedge C_{j_{1}} \wedge \cdots \wedge C_{j_{t}}=$ 0 for all $J$ if and only if $g-s-t+l>g-t$, which is equivalent to $l>s$. It follows that $\theta$ is in $G_{\mathbf{Q}}^{l}$, by Proposition 2.5.

Corollary 2.7. Let $G^{l} \subset H^{p}(J ; \mathbf{Z})$ be the subgroup generated by all l-good $p$ forms and let $G_{\mathbf{Q}}^{l}$ be the vector space $G^{l} \otimes_{\mathbf{Z}} \mathbf{Q}$. Let $O_{l} \subset H^{p}(J ; \mathbf{Z})$ be the subgroup generated by all l-orthogonal p-forms.

Then $O_{l} \otimes_{\mathbf{Z}} \mathbf{Q}=G_{\mathbf{Q}}^{l}=\operatorname{ker} L^{g-p+l}$.

Corollary 2.8. Let $\theta_{1}, \ldots, \theta_{p}$ be linearly independent integral harmonic 1-forms on $J$ and let $\theta=\theta_{1} \wedge \cdots \wedge \theta_{p}$ be the corresponding nonzero p-form on $J$. Let $\theta=\sum_{r=0}^{[p / 2]} L^{r}\left(\theta^{(r)}\right)$ be the Lefschet $z$ decomposition of $\theta$, where $\theta^{(r)}$ is a primitive $(p-2 r)$-form on $J$ and $L^{r}$ is the rth power of the Lefschetz operator.

Then $\theta$ is l-good if and only if $\theta^{(l)}=\cdots=\theta^{([p / 2])}=0$.

Proof. Writing $\theta$ in its Lefschetz decomposition and applying $L^{g-p+l}$, we see that all the terms with $0 \leq r \leq l-1$ are zero since $\theta^{(r)}$ is a primitive $(p-2 r)$ form. Since $L^{g-p+l}(\theta)$ is zero and the Lefschetz decomposition is unique, we must have that $\theta^{(r)}=0$ for $l \leq r \leq\left[\frac{p}{2}\right]$.

We remark that this corollary provides the equivalence of the two filtrations of $H^{p}(J ; \mathbf{R})$ described in the introduction. Also, for $l=1, g-p+l=g-p+1$, 
and it follows that $G_{\mathbf{Q}}^{1}$ consists precisely of all primitive $p$-forms. This result is given in [BH1] for $p=3$. However, for $p \geq 5$, the space $G_{\mathbf{Q}}^{[(p-1) / 2]}$ strictly contains all primitive $p$-forms.

We also remark that it is not true that $\theta=\theta_{1} \wedge \cdots \wedge \theta_{p}$ is $l$-good if and only if for some $(p-l+1)$ of the $\theta_{i}$ 's we have $\theta_{i} \cdot \theta_{j}=0$. As an example, take $p=5$ and $l=2$, and let $X$ be a Riemann surface of genus 5 with symplectic basis $A_{1}, \ldots, A_{5}, B_{1}, \ldots, B_{5}$ for $H^{1}(X ; \mathbf{Z})$. Let

$$
\begin{aligned}
& \theta_{1}=A_{1}, \quad \theta_{2}=B_{1}+A_{2}, \quad \theta_{3}=2 B_{1}+B_{2}+A_{3}, \\
& \theta_{4}=B_{1}+B_{2}+B_{3}+A_{4}, \quad \theta_{5}=-B_{2}-3 B_{1}+B_{3}+2 B_{4} .
\end{aligned}
$$

Then, we have $\theta_{i} \cdot \theta_{j} \neq 0$ if $i \neq j$, but $\theta_{1} \wedge \cdots \wedge \theta_{5}$ is good, since

$$
\theta_{1} \wedge \cdots \wedge \theta_{5}=\theta_{1}^{\prime} \wedge \cdots \wedge \theta_{5}^{\prime}
$$

where

$$
\begin{aligned}
& \theta_{1}^{\prime}=\theta_{1}, \quad \theta_{2}^{\prime}=\theta_{2}, \quad \theta_{3}^{\prime}=\theta_{1}-2 \theta_{2}+\theta_{3}, \\
& \theta_{4}^{\prime}=\theta_{1}-\theta_{2}+\theta_{4}, \quad \theta_{5}^{\prime}=-\theta_{1}+3 \theta_{2}+\theta_{5},
\end{aligned}
$$

as is easily checked, and this lies in $O_{2} \subset G^{2}$ since $\theta_{i}^{\prime} \cdot \theta_{j}^{\prime}=0$ for $2 \leq i<j \leq 5$. The following proposition tells us that this is the only type of counterexample.

Proposition 2.9. A decomposable integral p-form $\theta=\theta_{1} \wedge \cdots \wedge \theta_{p}$ on $J$ is $l$ good if and only if there exist integral harmonic 1-forms $\theta_{1}^{\prime}, \ldots, \theta_{p}^{\prime}$ such that $\theta=\theta_{1}^{\prime} \wedge \cdots \wedge \theta_{p}^{\prime}$ and $\theta_{i}^{\prime} \cdot \theta_{j}^{\prime}=0$ for $1 \leq i, j \leq p-l+1$. Hence, $O_{l}=G^{l}$.

Proof. If $\theta=\theta_{1}^{\prime} \wedge \cdots \wedge \theta_{p}^{\prime}$ is a decomposable integral $(p-l+1)$-form with $\theta_{i}^{\prime} \cdot \theta_{j}^{\prime}=0$ for all $1 \leq i, j \leq p-l+1$, we consider $\theta \widetilde{\cap} \eta^{l}$. As remarked after Lemma 2.2, pseudocap product with $\eta^{l}$ removes $l$ pairs of 1-forms, takes their pairwise product under the skew-symmetric bilinear form, and takes the product of these $l$ numbers as the coefficient for the wedge product of the remaining $(p-2 l) 1$-forms. Then it is clear that $\theta \widetilde{n} \eta^{l}=0$ since any choice of $l$ pairs of 1 -forms must contain $(l+1)$ mutually orthogonal 1 -forms, so one pair must consist of orthogonal 1-forms. Hence, all the coefficients are zero and $\theta$ is $l$-good, by Proposition 2.3. So $O_{l} \subset G^{l}$.

Suppose $\theta=\theta_{1} \wedge \cdots \wedge \theta_{p}$ is $l$-good. Let $\Pi$ be the oriented $p$-plane in $H^{1}(X ; \mathbf{Q})$ spanned by $\theta_{1}, \ldots, \theta_{p}$. Using a basic linear algebra result, we may choose a basis $\tau_{1}, \ldots, \tau_{s}, \tau_{1}^{\prime}, \ldots, \tau_{t}^{\prime}$ for $\Pi$ with $t \leq s, t+s=p, \tau_{i} \cdot \tau_{j}=0$ for $1 \leq i, j \leq s$, and $\tau_{i} \cdot \tau_{j}^{\prime}=n_{i} \delta_{i j}$ for some natural numbers $n_{i}$ with $n_{i}$ dividing $n_{i+1}$, which is equivalent over the integers to $\theta_{1}, \ldots, \theta_{p}$.

We remark that $s \geq\left[\frac{p-1}{2}\right]+1$ and $\theta= \pm \tau_{1} \wedge \cdots \wedge \tau_{s} \wedge \tau_{1}^{\prime} \wedge \cdots \wedge \tau_{t}^{\prime}$ since any integral change of basis has determinant \pm 1 .

If $s \geq p-l+1$, we are done by letting $\theta_{i}^{\prime}=\tau_{i}$ for $1 \leq i \leq s$ and $\theta_{i}^{\prime}=\tau_{i-s}^{\prime}$ for $s+1 \leq i \leq p$; and reversing $\tau_{s-1}$ and $\tau_{s}$, if necessary, to make $\theta=\theta_{1}^{\prime} \wedge \cdots \wedge \theta_{p}^{\prime}$.

Suppose $s<p-l+1$. We complete the basis $\tau_{1}, \ldots, \tau_{s}, \tau_{1}^{\prime}, \ldots, \tau_{t}^{\prime}$ for $\Pi$ to a basis $\tau_{1}, \ldots, \tau_{g}, \tau_{1}^{\prime}, \ldots, \tau_{g}^{\prime}$ for $H^{1}(X ; \mathbf{Q})$ such that $\tau_{i} \cdot \tau_{j}=0$ for $1 \leq i, j \leq g$, and $\tau_{i} \cdot \tau_{j}^{\prime}=n_{i} \delta_{i j}$ for some natural numbers $n_{i}$. We remark that we may choose $n_{i}=1$ if $i>t$. With respect to this basis, the Kaehler form $\Omega$ may be written $\Omega=\sum_{i=1}^{g} \frac{1}{n_{i}} \tau_{i}^{\prime} \wedge \tau_{i}$, so

$$
\frac{\Omega^{g-p+l}}{(g-p+l) !}=\sum_{I} N_{I} \tau_{i_{1}}^{\prime} \wedge \tau_{i_{1}} \wedge \cdots \wedge \tau_{i_{g-p+l}}^{\prime} \wedge \tau_{i_{g-p+l}},
$$


where the sum is over all multi-indices $I=\left(i_{1}, \ldots, i_{g-p+l}\right)$ with $1 \leq i_{1}<$ $\cdots<i_{g-p+l} \leq g$, and $N_{I}=1 / n_{i_{1}} \cdots n_{i_{g-p+l}}$.

So, $L^{g-p+l}(\theta) /(g-p+l)$ ! equals

$$
\pm \sum_{I} N_{I} \tau_{i_{1}} \wedge \tau_{i_{1}}^{\prime} \wedge \cdots \wedge \tau_{i_{g-p+l}} \wedge \tau_{i_{g-p+l}}^{\prime} \wedge \tau_{1} \wedge \cdots \wedge \tau_{s} \wedge \tau_{1}^{\prime} \wedge \cdots \wedge \tau_{t}^{\prime}
$$

We note that all nonzero terms in this expression are linearly independent and the only nonzero terms occur when $\left\{i_{1}, \ldots, i_{g-p+l}\right\} \subset\{s+1, \ldots, g\}$.

But the cardinality of the set $\{s+1, \ldots, g\}$ is $g-s>g-(p-l+1)=g-p+$ $l-1$, so $g-s \geq g-p+l$. Hence, we may choose a subset $\left\{i_{1}, \ldots, i_{g-p+l}\right\} \subset$ $\{s+1, \ldots, g\}$ and guarantee that $L^{g-p+l}(\theta) \neq 0$. This contradiction shows $s \geq p-l+1$, so that $G^{l} \subset O_{l}$, which proves the proposition.

We conclude this section by summarizing our results in the following theorem:

Theorem 2.10. Let $X$ be a Riemann surface of genus $g \geq p \geq 3$ and let $l$ be a natural number with $1 \leq l \leq\left[\frac{p-1}{2}\right]$. Let $G^{l}$ be the abelian subgroup of $H^{p}(J ; \mathbf{Z})$ generated by l-good p-forms. Let $O_{l}$ be the abelian subgroup of $H^{p}(J ; Z)$ generated by l-orthogonal p-forms. Let $L$ be the Lefschetz operator on $H^{p}(J ; \mathbf{Q})$ defined by taking the wedge product with the fundamental 2-form on $J$.

Then $O_{l}=G^{l}$. Further, if we consider the vector spaces generated by these abelian groups in $H^{p}(J ; \mathbf{Q})$ then $O_{l} \otimes_{\mathbf{Z}} \mathbf{Q}=G_{\mathbf{Q}}^{l}=\operatorname{ker} L^{g-p+l}$.

Moreover, suppose $\theta$ is a decomposable integral p-form and

$$
\theta=\sum_{r=0}^{[p / 2]} L^{r}\left(\theta^{(r)}\right)
$$

is the Lefschetz decomposition of $\theta$, where $\theta^{(r)}$ is a primitive $(p-2 r)$-form on $J$ and $L^{r}$ is the rth power of the Lefschetz operator.

Then $\theta$ is l-good if and only if $\theta^{(l)}=\cdots=\theta^{([p / 2])}=0$.

\section{HARMONIC volume}

In this section we will define harmonic volume and show that twice the value of harmonic volume on primitive $(2 k+1)$-forms equals the linear functional $\nu$. This relationship will enable us to utilize harmonic volume as a tool for calculating the image of the algebraic $k$-cycle $W_{k}-W_{k}^{-}$in the intermediate Jacobian $\mathscr{J}(J(X))$. In particular, we show that the values of harmonic volume on good forms in $G^{k-1}$ lie in a discrete subgroup of $\mathbf{R} / \mathbf{Z}$ and this will enable us in the next section to show that harmonic volume is identically zero on this class of good forms. by

Let $\theta=\theta_{1} \wedge \cdots \wedge \theta_{2 k+1}$ be a good $(2 k+1)$-form. Define $J_{k}: X_{k} \rightarrow T^{2 k+1}$

$$
J_{k}\left(x_{1}, \ldots, x_{k}\right)=\left(\sum_{i=1}^{k} \int_{p}^{x_{i}} \theta_{1}, \ldots, \sum_{i=1}^{k} \int_{p}^{x_{i}} \theta_{2 k+1}\right) \text { modulo } \mathbf{Z}^{2 k+1}
$$

where $p$ is a fixed basepoint in $X$. We know from $\S 2$ that $J_{k} X_{k}$, as a singular $2 k$-cycle, is the boundary of some $(2 k+1)$-chain, $C_{2 k+1}$, in $T^{2 k+1}$. We define 
the harmonic volume of $\theta, I(\theta)$, to be

$$
I(\theta)=\int_{C_{2 k+1}} d x_{1} \wedge \cdots \wedge d x_{2 k+1} \text { modulo } \mathbf{Z},
$$

where $\theta_{i}$ is the pullback of $d x_{i}$ by the Abel-Jacobi map.

We remark that if we change the basepoint in $X$ used to define the map $J_{k}$, the map is changed by translation. Since integration is invariant under rigid translation, the mapping $I$ is independent of the basepoint in $X$. We also remark that for a hyperelliptic Riemann surface $X$, harmonic volume vanishes identically, just in the case $k=1$.

Our first result is to demonstrate harmonic volume as a sum of integrals over a nested sequence of submanifolds of the symmetric product $X_{k}$. This result is analogous to Harris' result representing harmonic volume as an iterated integral.

Proposition 3.1. Let $\theta=\theta_{1} \wedge \cdots \wedge \theta_{2 k+1}$ be a nonzero good $(2 k+1)$-form. Let $N$ be the largest integer so that $\theta_{N} \wedge \cdots \wedge \theta_{2 k+1}$ is exact on $X_{k}$. We remark that $2 \leq N \leq 2 k$. Let $\gamma_{2 k}=X_{k}$, and for each $j, N-1 \leq j \leq 2 k-1$, let $\gamma_{j}$ be a j-manifold in $\gamma_{j+1}$ dual to $\theta_{j+2}$. Let $f_{j}(x)=\int_{p}^{x} \theta_{j}$. Then there exist forms $\eta_{1, \ldots, N} ; \ldots ; \eta_{1, \ldots, 2 k}$ with each $\eta_{1, \ldots, j}$ being $a(j-1)$-form on $\gamma_{j}$ and satisfying

$$
d \eta_{1, \ldots, j}=J_{k}^{*}\left(d x_{1} \wedge \cdots \wedge d x_{j}\right) \text { and } \int_{\gamma_{j}} \eta_{1, \ldots, j} \wedge \theta_{j+1}=0
$$

Further,

$$
\begin{aligned}
I(\theta)= & (-1)^{N-1} \int_{\gamma_{N-1}} f_{N} \theta_{1} \wedge \cdots \wedge \theta_{N-1}+(-1)^{N} \eta_{1, \ldots, N} \\
& -\int_{\gamma_{N}} \eta_{1, \ldots, N+1}-\cdots-\int_{\gamma_{2 k-1}} \eta_{1, \ldots, 2 k} \quad \text { modulo } \mathbf{Z},
\end{aligned}
$$

and the value of the integral is independent of the choice of the $\eta_{1, \ldots, j}$ 's. Moreover, if $\tilde{\gamma}_{j}$ denotes $\gamma_{j}$ cut along the submanifold $\gamma_{j-1}$, then

$$
\begin{aligned}
I(\theta)= & \int_{\widetilde{X}_{k}} f_{2 k+1} \theta_{1} \wedge \cdots \wedge \theta_{2 k}-\int_{\tilde{\gamma}_{2 k-1}} f_{2 k} \theta_{1} \wedge \cdots \wedge \theta_{2 k-1} \\
& +\cdots+(-1)^{N-1} \int_{\gamma_{N-1}} f_{N} \theta_{1} \wedge \cdots \wedge \theta_{N-1} \quad \text { modulo } \mathbf{Z} .
\end{aligned}
$$

Proof. Let $\gamma_{n}$ be an oriented $n$-manifold, $n \geq 2$, and let $\theta_{1}, \ldots, \theta_{n+1}$ be $n+1$ closed 1-forms on $\gamma_{n}$ with integer periods so that $\theta_{n+1}$ is not exact on $\gamma_{n}$. Let $p \in \gamma_{n}$ be a fixed basepoint and suppose also that the image of the map $\boldsymbol{\Theta}_{n}: \gamma_{n} \rightarrow T^{n+1}$ given by

$$
x \mapsto\left(\int_{p}^{x} \theta_{1}, \ldots, \int_{p}^{x} \theta_{n+1}\right) \quad \text { modulo } \mathbf{Z}^{n+1}
$$

is the boundary of some $(n+1)$-chain in $T^{n+1}$.

Let $\gamma_{n-1}$ be an $(n-1)$-manifold in $\gamma_{n}$ dual to $\theta_{n+1}$. Let $\tilde{\gamma}_{n}$ be $\gamma_{n}$ cut along $\gamma_{n-1}$ so that $\partial \tilde{\gamma}_{n}=\gamma_{n-1}^{+} \cup \gamma_{n-1}^{-}$. Then $f_{n+1}(x)=\int_{p}^{x} \theta_{n+1}$ is well defined on $\tilde{\gamma}_{n}$ with $f_{n+1}\left(p^{-}\right)=f_{n+1}\left(p^{+}\right)+1$, where $p^{+} \in \gamma_{n-1}^{+}$and $p^{-} \in \gamma_{n-1}^{-}$correspond to $p \in \gamma_{n-1}$. 
We have

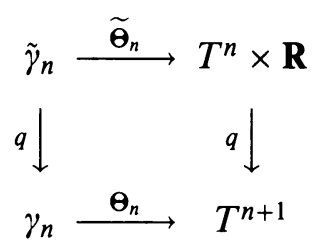

where $q$ are the natural quotient maps. Note that the oriented boundary of $\tilde{\Theta}_{n}\left(\tilde{\gamma}_{n}\right)$ is $(-1)^{n} \tilde{\Theta}_{n}\left(\gamma_{n-1}^{+}\right) \cup(-1)^{n-1} \widetilde{\Theta}_{n}\left(\gamma_{n-1}^{-}\right)$.

Let $1 \leq i_{1}<\cdots<i_{n-1} \leq n$. Then, we have

$$
\begin{aligned}
\int_{\widetilde{\boldsymbol{\Theta}}_{n}\left(\gamma_{n-1}\right)} d x_{i_{1}} \wedge \cdots \wedge d x_{i_{n-1}}=\int_{\gamma_{n-1}} d\left(\boldsymbol{\Theta}_{n}\right)_{i_{1}} \wedge \cdots \wedge d\left(\boldsymbol{\Theta}_{n}\right)_{i_{n-1}} \\
=\int_{\gamma_{n-1}} \theta_{i_{1}} \wedge \cdots \wedge \theta_{i_{n-1}} \\
=\int_{\gamma_{n}} \theta_{i_{1}} \wedge \cdots \wedge \theta_{i_{n-1}} \wedge \theta_{n+1} \\
=\int_{\boldsymbol{\Theta}_{n}\left(\gamma_{n}\right)} d x_{i_{1}} \wedge \cdots \wedge d x_{i_{n-1}} \wedge d x_{n+1} \\
=0
\end{aligned}
$$

by Stokes' theorem, since $\boldsymbol{\theta}_{n}\left(\gamma_{n}\right)$ is a boundary.

Since

$$
\int_{\widetilde{\boldsymbol{\Theta}}_{n}\left(\gamma_{n-1}\right)} d x_{i_{1}} \wedge \cdots \wedge d x_{i_{n-1}}=0
$$

for all sequences $1 \leq i_{1}<\cdots<i_{n-1} \leq n, \widetilde{\Theta}_{n}\left(\gamma_{n-1}^{+}\right)$is the boundary of some $n$-chain, $D_{n}^{+}$, in $T^{n} \times \mathbf{R}$. Then $\widetilde{\Theta}_{n}\left(\gamma_{n-1}^{-}\right)=\partial D_{n}^{-}$, where $D_{n}^{-}=D_{n}^{+}+$ $(0, \ldots, 0,1)$. Let $Y=\widetilde{\Theta}_{n}\left(\tilde{\gamma}_{n}\right)-(-1)^{n}\left(D_{n}^{+}-D_{n}^{-}\right)$, where addition is as singular chains. Then $Y$ is an $n$-cycle in $T^{n} \times \mathbf{R}$ and under the quotient map, $q, q(Y)=$ $\boldsymbol{\theta}_{n}\left(\gamma_{n}\right)$.

Since the map on homology induced by the quotient map $q: T^{n} \times \mathbf{R} \rightarrow T^{n+1}$ is injective and $q(Y)$ is a boundary in $T^{n+1}$, we conclude that $Y$ is a boundary in $T^{n} \times \mathbf{R}$. Say $Y=\partial C_{n+1}$, where $C_{n+1}$ is a $(n+1)$-chain in $T^{n} \times \mathbf{R}$. Then $\partial q\left(C_{n+1}\right)=\boldsymbol{\Theta}_{n}\left(\gamma_{n}\right)$, so,

$$
\begin{aligned}
& \int_{q\left(C_{n+1}\right)} d x_{1} \wedge \cdots \wedge d x_{n+1} \\
&=\int_{C_{n+1}} d x_{1} \wedge \cdots \wedge d x_{n+1} \\
&=(-1)^{n} \int_{Y} x_{n+1} d x_{1} \wedge \cdots \wedge d x_{n} \\
&=(-1)^{n} \int_{\widetilde{\Theta}_{n} \tilde{\gamma}_{n}} x_{n+1} d x_{1} \wedge \cdots \wedge d x_{n} \\
&-\int_{D_{n}^{+}} x_{n+1} d x_{1} \wedge \cdots \wedge d x_{n}+\int_{D_{n}^{-}} x_{n+1} d x_{1} \wedge \cdots \wedge d x_{n} \\
&=(-1)^{n} \int_{\tilde{\gamma}_{n}} f_{n+1} \Theta_{n}^{*}\left(d x_{1} \wedge \cdots \wedge d x_{n}\right)+\int_{D_{n}} d x_{1} \wedge \cdots \wedge d x_{n}
\end{aligned}
$$


We remark that we have used the commutative diagram ( $\dagger$ ) to identify $\widetilde{\Theta}_{n}^{*}\left(d x_{1} \wedge \cdots \wedge d x_{n}\right)$ and $\Theta_{n}^{*}\left(d x_{1} \wedge \cdots \wedge d x_{n}\right)$ by means of the quotient map $q$, where $d x_{1} \wedge \cdots \wedge d x_{n}$ is considered as a form on $T^{n} \times \mathbf{R}$ and $T^{n+1}$, respectively.

It is easily computed that

$$
\int_{\tilde{\gamma}_{n}} \tilde{\boldsymbol{\Theta}}_{n}^{*}\left(d x_{1} \wedge \cdots \wedge d x_{n}\right)=\int_{\gamma_{n}} \boldsymbol{\Theta}_{n}^{*}\left(d x_{1} \wedge \cdots \wedge d x_{n}\right)=\int_{\boldsymbol{\Theta}_{n}\left(\gamma_{n}\right)} d x_{1} \wedge \cdots \wedge d x_{n}=0,
$$

since $\boldsymbol{\Theta}_{n}\left(\gamma_{n}\right)$ is a boundary, and it follows that there exists a $(n-1)$-form $\eta_{1, \ldots, n}$ on $\gamma_{n}$ such that

$$
d \eta_{1, \ldots, n}=\Theta_{n}^{*}\left(d x_{1} \wedge \cdots \wedge d x_{n}\right) .
$$

Suppose $\int_{\gamma_{n}} \psi \wedge \theta_{n+1}=0$ for all closed $(n-1)$-forms $\psi$ on $\gamma_{n}$. It then follows that $\theta_{n+1}$ is exact on $\gamma_{n}$, contradicting the hypothesis that this form is not exact. Thus, there exists a closed $(n-1)$-form $\psi$ on $\gamma_{n}$ with

$$
\int_{\gamma_{n}} \psi \wedge \theta_{n+1}=1 \text {. }
$$

Say

$$
\int_{\gamma_{n}} \eta_{1, \ldots, n} \wedge \theta_{n+1}=C
$$

We then replace $\eta_{1, \ldots, n}$ by $\eta_{1, \ldots, n}-C \psi$ and obtain a new $(n-1)$-form, which we will still call $\eta_{1, \ldots, n}$, on $\gamma_{n}$ such that

$$
d \eta_{1, \ldots, n}=\mathbf{\Theta}_{n}^{*}\left(d x_{1} \wedge \cdots \wedge d x_{n}\right) \text { and } \int_{\gamma_{n}} \eta_{1, \ldots, n} \wedge \theta_{n+1}=0 .
$$

Then

$$
\begin{gathered}
\int_{\tilde{\gamma}_{n}} f_{n+1} \Theta_{n}^{*}\left(d x_{1} \wedge \cdots \wedge d x_{n}\right)=\int_{\tilde{\gamma}_{n}} f_{n+1} d \eta_{1, \ldots, n} \\
\quad=\int_{\tilde{\gamma}_{n}} f_{n+1} d \eta_{1, \ldots, n}+\theta_{n+1} \wedge \eta_{1, \ldots, n} \\
=\int_{\tilde{\gamma}_{n}} d\left(f_{n+1}, \eta_{1, \ldots, n}\right) \\
=\int_{(-1)^{n} \gamma_{n-1}^{+} \cup(-1)^{n+1} \gamma_{n-1}^{-}} f_{n+1} \eta_{1, \ldots, n} \\
=(-1)^{n+1} \int_{\gamma_{n-1}} \eta_{1, \ldots, n} .
\end{gathered}
$$

Note that if we choose another $(n-1)$-form $\eta_{1, \ldots, n}^{\prime}$ on $\gamma_{n}$ satisfying $(*)$, then $\eta_{1, \ldots, n}-\eta_{1, \ldots, n}^{\prime}$ is a closed $(n-1)$-form on $\gamma_{n}$, so

$$
\int_{\gamma_{n-1}} \eta_{1, \ldots, n}-\eta_{1, \ldots, n}^{\prime}=\int_{\gamma_{n}}\left(\eta_{1, \ldots, n}-\eta_{1, \ldots, n}^{\prime}\right) \wedge \theta_{n+1}=0,
$$

so the choice of $\eta_{1, \ldots, n}$ is irrelevant.

So,

$$
\int_{C_{n+1}} d x_{1} \wedge \cdots \wedge d x_{n+1}=\int_{D_{n}} d x_{1} \wedge \cdots \wedge d x_{n}-\int_{\gamma_{n-1}} \eta_{1, \ldots, n}
$$


The $n$-form $d x_{1} \wedge \cdots \wedge d x_{n}$ on $T^{n} \times \mathbf{R}$ arises by pulling back the form $d x_{1} \wedge \cdots \wedge d x_{n}$ on $T^{n}$ by the projection $p: T^{n} \times \mathbf{R} \rightarrow T^{n}$. Hence, if $D_{n}^{\#}=$ $p_{\#}\left(D_{n}\right)$, we have

$$
\int_{D_{n}} d x_{1} \wedge \cdots \wedge d x_{n}=\int_{D_{n}^{*}} d x_{1} \wedge \cdots \wedge d x_{n} .
$$

Note that $\partial D_{n}^{\#}=p_{\#}\left(\partial D_{n}\right)=p_{\#}\left(\tilde{\boldsymbol{\Theta}}_{n}\left(\gamma_{n-1}\right)\right)$ is the image of $\gamma_{n-1}$ under the map $\boldsymbol{\Theta}_{n-1}: \gamma_{n-1} \rightarrow T^{n}$, given by integrating $\theta_{1}, \ldots, \theta_{n}$ modulo $\mathbf{Z}$ along a path.

Now $\gamma_{n-1}$ is an oriented $(n-1)$-manifold whose image under the map $\boldsymbol{\theta}_{n-1}$, defined by integrating $\theta_{1}, \ldots, \theta_{n}$ along a path, is the boundary of some $n$-chain in $T^{n}$. If the form $\theta_{n}$ is not exact on $\gamma_{n-1}$, then we have an inductive step.

Thus, we obtain

$$
\begin{aligned}
I(\theta)= & \int_{D_{N}^{*}} d x_{1} \wedge \cdots \wedge d x_{N}-\int_{\gamma_{N-1}} \eta_{1, \ldots, N} \\
& -\cdots-\int_{\gamma_{2 k-1}} \eta_{1, \ldots, 2 k} \quad \text { modulo } \mathbf{Z},
\end{aligned}
$$

where $\gamma_{2 k}=X_{k}$ and $\gamma_{l}$ is an $l$-manifold in $\gamma_{l+1}$ dual to $\theta_{l+2} ; D_{N}^{\#}$ is an $N$-chain in $T^{N}$ whose boundary is the image of the map $\Theta_{N-1}: \gamma_{N-1} \rightarrow T^{N}$ given by integrating $\theta_{1}, \ldots, \theta_{N}$ modulo $\mathbf{Z}$ along a path; and $\eta_{1, \ldots, l}$ is an $(l-1)$-form on $\gamma_{l}$ with

$$
d \eta_{1, \ldots, l}=J_{k}^{*}\left(d x_{1} \wedge \cdots \wedge d x_{l}\right) \text { and } \int_{\gamma_{l}} \eta_{1, \ldots, l} \wedge \theta_{l+1}=0 .
$$

Since $\theta_{N}$ is exact on $\gamma_{N-1}$, the mapping $\Theta_{N-1}$ lifts to a mapping $\widehat{\Theta}_{N-1}$ with the following diagram commuting:

$$
\begin{array}{cc}
\gamma_{N-1} \stackrel{\widehat{\boldsymbol{\Theta}}_{N-1}}{\longrightarrow} T^{N-1} \times \mathbf{R} \\
q \downarrow \\
\gamma_{N-1} \stackrel{\boldsymbol{\Theta}_{N-1}}{\longrightarrow} T^{N}
\end{array}
$$

The injectivity of the map induced on homology by $q: T^{N-1} \times \mathbf{R} \rightarrow T^{N}$ yields that $\widehat{\boldsymbol{\Theta}}_{N-1}\left(\gamma_{N-1}\right)$ is a boundary in $T^{N-1} \times \mathbf{R}$. If $\bar{D}_{N}$ is an $N$-chain in $T^{N-1} \times \mathbf{R}$ with boundary $\widehat{\Theta}_{N-1}\left(\gamma_{N-1}\right)$, then

$$
\int_{D_{N}^{*}} d x_{1} \wedge \cdots \wedge d x_{N} \equiv \int_{\bar{D}_{N}} d x_{1} \wedge \cdots \wedge d x_{N} \quad \text { modulo } \mathbf{Z},
$$

since $q\left(\bar{D}_{N}\right)$ and $D_{N}^{\#}$ differ by a cycle in $T^{N}$.

But, in $T^{N-1} \times \mathbf{R}, d x_{1} \wedge \cdots \wedge d x_{N}=(-1)^{N-1} d\left(x_{N} d x_{1} \wedge \cdots \wedge d x_{N-1}\right), \quad$ so

$$
\begin{gathered}
\int_{D_{N}^{*}} d x_{1} \wedge \cdots \wedge d x_{N} \equiv \int_{\bar{D}_{N}} d x_{1} \wedge \cdots \wedge d x_{N} \quad \text { modulo } \mathbf{Z} \\
=(-1)^{N-1} \int_{\widehat{\Theta}_{N-1}\left(\gamma_{N-1}\right)} x_{N} d x_{1} \wedge \cdots \wedge d x_{N-1} \\
=(-1)^{N-1} \int_{\gamma_{N-1}} f_{N} \theta_{1} \wedge \cdots \wedge \theta_{N-1}
\end{gathered}
$$

This serves to complete the proof. 
Our next result is the crucial connection between harmonic volume and the image of $W_{k}-W_{k}^{-}$in the intermediate Jacobian $\mathscr{J}(J(X))$.

Proposition 3.2. On good forms, the homomorphism $\nu$ defined by integration over a chain whose boundary is $W_{k}-W_{k}^{-}$, equals twice the harmonic volume. That is, $\nu=2 I$.

Proof. By defining $j: H^{1}(X ; \mathbf{R}) \rightarrow H^{1}(X ; \mathbf{R})$ by $j(\alpha)=-{ }^{*} \alpha$, where $*$ denotes the Hodge star operator, we induce a complex structure on $H^{1}(X ; \mathbf{R})$ which makes it isomorphic to $H^{1,0}(X)$, so we may consider the Jacobian $J$ to be $\left[H^{1}(X ; \mathbf{R})\right]^{*}$ modulo the lattice generated by periods of $\theta_{1}, \ldots, \theta_{g},{ }^{*} \theta_{1}$, $\ldots,{ }^{*} \theta_{g}$.

Consider the commutative diagram

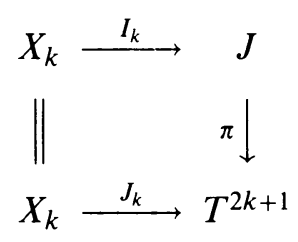

where $J_{k}$ is the map defined above by integrating $\theta_{1}, \ldots, \theta_{2 k+1}$ along a path; and $\pi$ is projection on the first $2 k+1$ factors.

If $D_{2 k+1}$ is a $(2 k+1)$-chain on $J$ with boundary $W_{k}-W_{k}^{-}$and $C_{2 k+1}$ is a chain on $T^{2 k+1}$ with boundary $J_{k} X_{k}$, then it is easily shown that $\pi_{\#} D_{2 k+1}$ and $C_{2 k+1}-i_{\#} C_{2 k+1}$, where $i$ is the group involution on $T^{2 k+1}$, are homologous. Now using this fact and the definition of $\nu$ and $I$, the result follows.

We now show that, for $k \geq 2$ and for an element in $G^{k-1}$, the image $\nu(\theta)$ lies in a discrete subgroup of $\mathbf{R} / \mathbf{Z}$. We will use this result in $\S 4$ to show that the homomorphism $\nu$ is identically zero on $G^{k-1}$.

Lemma 3.3. For $k \geq 2$ and $\theta=\theta_{1} \wedge \cdots \wedge \theta_{2 k+1}$ a generator of $O_{k-1}$, as defined in $\S 2$, then $\nu(\theta)$ is a ( $k !)$-torsion point in $\mathbf{R} / \mathbf{Z}$. Consequently, if $\theta$ is a decomposable integral $(2 k+1)$-form in the kernel of the $(g-k-2)$ th power of the Lefschetz operator, then $\theta$ is $(k-1)$-good and $\nu(\theta)$ is a (k!)-torsion point in $\mathbf{R} / \mathbf{Z}$.

Proof. Rather than considering $W_{k}$, the image of $X_{k}$ in $J$, consider $m_{\#} W_{1}^{k}$, the image of $X^{k}$ in $J$, where $m: J \times \cdots \times J \rightarrow J$ is multiplication.

We write $W_{1}^{k}-\left(W_{1}^{-}\right)^{k}$ as the sum

$$
m_{\#}\left(W_{1}^{k}-\left(W_{1}^{-}\right)^{k}\right)=\sum_{j=0}^{k-1} m_{\#}\left[\left(W_{1}-W_{1}^{-}\right) \times W_{1}^{k-j-1} \times\left(W_{1}^{-}\right)^{j}\right] .
$$

Now, choosing a 3-chain $D_{3}$ whose boundary is $W_{1}-W_{1}^{-}$, then the cycle $m_{\#}\left(W_{1}^{k}-\left(W_{1}^{-}\right)^{k}\right)$ is the boundary of

$$
D_{2 k+1}=\sum_{j=0}^{k-1} m_{\#}\left[D_{3} \times W_{1}^{k-j-1} \times\left(W_{1}^{-}\right)^{j}\right] .
$$


Using the fact that $k ! \nu(\theta)=\int_{D_{2 k+1}} \theta$ and the fact that $\theta$ is in $O_{k-1}$ and therefore at least $k+3$ of the $\theta_{i}$ 's are orthogonal, a simple computation of $\int_{D_{2 k+1}} \theta$ completes the proof.

We summarize the results from this section in the following theorem:

Theorem 3.4. Let $X$ be a nonsingular algebraic curve of genus $g \geq 2 k+1$ for a fixed natural number $k$. Let $J=J(X)$ be the Jacobian variety of $X$. Let $I$ denote the harmonic volume map and let $\nu$ denote the map on integral $(2 k+1)$ forms given by integration modulo $\mathbf{Z}$ over a chain $D_{2 k+1}$ whose boundary is the $k$-cycle $W_{k}-W_{k}^{-}$. Let $\tilde{\nu}$ be the associated vector space map, which is well-defined modulo periods. Then the $\mathbf{C}$-linear map $\tilde{\nu}$ is identically zero on $(r, 2 k+1-r)$-forms if $r<\left[\frac{k}{2}\right]$ or if $r>2 k+1-\left[\frac{k}{2}\right]$. Further, harmonic volume is a well-defined map which is independent of the basepoint in $X$ used to define the map $J_{k}$. Proposition 3.1 presents a formula demonstrating harmonic volume as sum of integrals over a nested sequence of submanifolds of $X_{k}$.

Further, when restricted to $k$-good $(2 k+1)$-forms, the homomorphism $\nu$ equals twice the harmonic volume. The image of $G^{k-1}$ under the homomorphism $\nu$ is contained in a discrete subgroup of $\mathbf{R} / \mathbf{Z}$. Recalling Proposition 1.1, if $W_{k}$ is algebraically equivalent to $W_{k}^{-}$, it is necessary for $\tilde{\nu}$ to vanish on forms of Hodge type $(r, 2 k+1-r)$ if $r \neq k$ and $r \neq k+1$.

\section{VARIATION OF HARMONIC VOLUME}

In this section we will use the interpretation of harmonic volume as the "volume" of a chain to utilize the methods of integral calculus in order to compute the change in harmonic volume as the complex structure on $X$ varies. The connection between harmonic volume and $\nu$ will then allow us to derive a formula for the "vertical codifferential" of $\nu$ when considered as a section of a holomorphic torus bundle.

Let $G$ be the free abelian group of good $(2 k+1)$-forms on $J$. Let $G_{\mathbf{R}}=$ $G \otimes_{\mathbf{Z}} \mathbf{R}$ and $G_{\mathbf{C}}=G_{\mathbf{R}} \otimes_{\mathbf{R}} \mathbf{C}$. We remark that $G_{\mathbf{R}}$ and $G_{\mathbf{C}}$ may be considered as vector subspaces of $H^{2 k+1}(J ; \mathbf{R})$ and $H^{2 k+1}(J ; \mathbf{C})$, respectively. By the Hodge Theorem, $H^{2 k+1}(J ; \mathbf{C})=\bigoplus_{p+q=2 k+1} H^{p, q}(J)$, and, letting $G^{p, q}=H^{p, q}(J) \cap$ $G_{\mathbf{C}}$, we get $G_{\mathbf{C}}=\bigoplus G^{p, q}$, since the Lefschetz operator is a real operator of bidegree $(1,1)$. As mentioned in the introduction, the Hodge filtration induces a filtration on the subspace of $H^{2 k+1}(J ; \mathbf{C})$ generated by good forms. The inclusion

$$
G_{\mathbf{R}} \rightarrow G_{\mathbf{C}} / \overline{F^{k+1} G_{\mathbf{C}}} \cong F^{k+1} G_{\mathbf{C}},
$$

gives $G_{\mathbf{R}}$ a natural complex structure that, by the work of Griffiths, varies holomorphically with the complex structure on $J$.

The group homomorphism can then be realized as a point on the torus

$$
\operatorname{Hom}_{\mathbf{C}}\left(F^{k+1} G_{\mathbf{C}}, \mathbf{C}\right) / \operatorname{Hom}_{\mathbf{Z}}(G, \mathbf{Z})
$$

in the following manner. The map $\nu$ is an element of $\operatorname{Hom}(G, \mathbf{R} / \mathbf{Z})$. Now, $\operatorname{Hom}(G, \mathbf{R} / \mathbf{Z})$ is isomorphic to the quotient $\operatorname{Hom}(G, \mathbf{R}) / \operatorname{Hom}(G, \mathbf{Z})$, and $\operatorname{Hom}(G, \mathbf{R})$ can be identified with $\operatorname{Hom}_{\mathbf{R}}\left(G_{\mathbf{R}}, \mathbf{R}\right)$. Further, if we define

$$
\mu: \operatorname{Hom}_{\mathbf{R}}\left(G_{\mathbf{R}}, \mathbf{R}\right) \rightarrow \operatorname{Hom}_{\mathbf{C}}\left(G_{\mathbf{R}}, \mathbf{C}\right)
$$


by

$$
\mu(\lambda)(p)=\lambda(j(p))+i \lambda(p),
$$

we then identify $\operatorname{Hom}_{\mathbf{R}}\left(G_{\mathbf{R}}, \mathbf{R}\right)$ with $\operatorname{Hom}_{\mathbf{C}}\left(G_{\mathbf{R}}, \mathbf{C}\right)$. Denoting $\operatorname{Hom}(G, \mathbf{Z})$ by $G^{*}$ and $\operatorname{Hom}_{\mathbf{C}}\left(F^{k+1} G_{\mathrm{C}}, \mathrm{C}\right)$ by $G_{+}^{*}$, we may consider $\nu$ as a point on the torus $G_{+}^{*} / G^{*}$, a compact complex Lie group similar to the intermediate Jacobian of the manifold $J$.

Now suppose we consider a holomorphic family of compact Riemann surfaces $X_{s}$, all of a fixed genus $g \geq 2 k+1$, where $s$ ranges over some complex open disk about the origin and $X_{0}=X$. Then we may consider the homology basis as fixed and the complex structure as varying holomorphically. Then the torus bundle on Torelli space with fiber $G_{+}^{*}(s) / G^{*}(s)$ is holomorphic and the linear functional $\nu$ provides a holomorphic section of this bundle. These results are essentially the work of Griffiths [G1, Proposition 3.8, G2, Theorems 1.1 and 1.27]. The holomorphic variation of $\nu$ implies the following result which generalizes the fact that $\nu$ vanishes on holomorphic differentials as shown in Proposition 1.2.

Proposition 4.1. For $k \geq 2$, the homomorphism $\nu$ is identically zero on $G^{k-1}$. Proof. Let $k \geq 2$ and let $X$ be a Riemann surface of genus $g \geq 2 k+1$. Consider $X$ as varying in Torelli space. That is, let $\Delta$ be a $(3 g-3)$-dimensional complex disk containing the origin with each $s \in \Delta$ corresponding to a Riemann surface $X_{s}$ so that $X_{0}=X$ and so that $\Delta$ is a complete, effective parametrization of the variation of complex structure of $X$.

Let $\theta=\theta_{1} \wedge \cdots \wedge \theta_{2 k+1}$ be a decomposable $(2 k+1)$-form on the Jacobian $J(X)$ which generates $O_{k-1}$ and let $\theta_{s}$ be a harmonic 1-form on $X_{s}$ representing the same cohomology class as $\theta$. Thus, $\theta_{s}$ is a $(k-1)$-good form on $J_{s}$ and by Lemma 3.3, $\nu_{s}\left(\theta_{s}\right)$ is a $\left(k\right.$ !)-torsion point in $\mathbf{R} / \mathbf{Z}$. Since $\nu_{s}$ varies analytically with $s$ and maps continuously into a discrete set, $\nu_{s}$ is constant on $\theta_{s}$ as $s$ varies. Now, choosing some $s_{0}$ for which the corresponding Riemann surface $X_{s_{0}}$ is hyperelliptic, and therefore $\nu_{s_{0}}$ is known to be identically zero, serves to conclude the proof.

Hence, the homomorphism $\nu$ is identically zero on primitive forms for $k \geq 2$. Hence, in order to gather any useful information about the algebraic equivalence of $W_{k}$ and $W_{k}^{-}$one must consider the larger class of good forms.

Now we consider the variation of harmonic volume as the complex structure of $X$ varies. To accomplish this, we will invoke the theory of deformation of complex structure as described in [SS]. For a more modern treatment, the reader is also referred to $[\mathrm{K}]$.

Fix a point $t$ in the Riemann surface $X$ and introduce a local coordinate $z$ which vanishes at $t$. Let $s=\rho^{2} e^{2 i \phi}$ be a complex number of sufficiently small modulus so that the closed disk $|z| \leq \rho$ lies in the image of the local coordinate $z$. Let $z^{*}$ be a local coordinate whose image contains an annular neighborhood $U$ of the curve $|z|=\rho$, but which omits a neighborhood of $t$. We define a new Riemann surface $X_{s}$ by removing the disk $|z|<\rho$ from $X$ and attaching a disk with local coordinate $z$ by identifying $z^{*}=z+\frac{s}{z}$ along the overlap $U \cap\{z|| z \mid \geq \rho\}$. Thus, $X_{0}=X$ and $X_{s}$ defines a holomorphic family of Riemann surfaces as $s$ varies over some small open disk. A Riemann surface $X^{*}$ is a deformation of $X$ if $X^{*}$ is conformal to $X_{s}$ for some $s$. 
We remark that if $\theta$ is a harmonic 1 -form on $X$ representing a cohomology class $[\theta]$ in $H^{1}(X ; \mathbf{R})$, then since $H^{1}(X ; \mathbf{R}) \cong H^{1}\left(X^{*} ; \mathbf{R}\right),[\theta]$ may also be considered as a cohomology class in $H^{1}(X ; \mathbf{R})$. However, $\theta$ may no longer be harmonic on $X^{*}$, but there is a unique harmonic 1 -form $\theta^{*}$ on $X^{*}$ with $\left[\theta^{*}\right]=[\theta]$. We say $\theta^{*}$ is the harmonic 1 -form on $X^{*}$ associated to $\theta$.

Choose $3 g-3$ generic points $t_{1}, \ldots, t_{3 g-3}$ on $X$ and perform this same deformation technique at each point $t_{j}$, making sure that the coordinate patches on which the deformations take place do not overlap. This gives a $(3 g-3)$ dimensional parameter space $\Delta$ with coordinates $s_{1}, \ldots, s_{3 g-3}$ over which the complex structure of $X$ varies. If the points $t_{1}, \ldots, t_{3 g-3}$ are chosen generically so that any quadratic differential vanishing at all these points vanishes identically, then the evaluation map

$$
\begin{gathered}
T_{s}(\Delta) \otimes H^{0}\left(X ; K^{2}\right) \rightarrow \mathbf{C}, \\
\sum_{j=1}^{3 g-3} c_{j} \frac{\partial}{\partial s_{j}} \otimes q(z)(d z)^{2} \mapsto \sum_{j=1}^{3 g-3} c_{j} q\left(t_{j}\right),
\end{gathered}
$$

is nondegenerate in the second factor, and it follows that the Kodaira-Spencer map taking $T_{s}(\Delta)$ to $H^{1}(X ; \Phi)$, where $\Phi=K^{-1}$, is surjective, hence an isomorphism. Hence, by the Kodaira-Spencer completeness theorem, the disk $\Delta$ is a complete, effective parametrization of all possible variations of the complex structure of $X$.

Let $X$ be a Riemann surface of genus $g \geq 2 k+1$ for a fixed natural number $k$ and let $\theta_{1}, \ldots, \theta_{2 k+1}$ be harmonic 1 -forms on $X$ so that $\theta_{1} \wedge \cdots \wedge \theta_{2 k+1}$ is a good $(2 k+1)$-form. Let $X^{*}$ be a deformation of $X$ and let $\theta_{1}^{*}, \ldots, \theta_{2 k+1}^{*}$ be the harmonic 1-forms on $X^{*}$ associated to $\theta_{1}, \ldots, \theta_{2 k+1}$. We remark that $\theta_{1}^{*} \wedge \cdots \wedge \theta_{2 k+1}^{*}$ is still a good $(2 k+1)$-form. Let $J_{k}: X_{k} \rightarrow T^{2 k+1}$ be defined as in $\S 2$. That is

$$
J_{k}\left(x_{1}, \ldots, x_{k}\right)=\left(\sum_{j=1}^{k} \int_{p}^{x_{j}} \theta_{1}, \ldots, \sum_{j=1}^{k} \int_{p}^{x_{j}} \theta_{2 k+1}\right) \quad \text { modulo } \mathbf{Z}^{2 k+1} \text {. }
$$

Likewise, let $J_{k}^{*}: X_{k}^{*} \rightarrow T^{2 k+1}$ be defined using $\theta_{1}^{*}, \ldots, \theta_{2 k+1}^{*}$.

Recall that the harmonic volume of $\theta, I(\theta)$, is the volume modulo $\mathrm{Z}$ of a $(2 k+1)$-chain in $T^{2 k+1}$ which is bounded by $J_{k} X_{k}$. Similarly, $I\left(\theta^{*}\right)$ is the volume modulo $\mathbf{Z}$ of a $(2 k+1)$-chain in $T^{2 k+1}$ which is bounded by $J_{k}^{*} X_{k}^{*}$. Thus, for $X^{*}$ near $X$, and hence for $X_{k}^{*}$ near $X_{k}, I\left(\theta^{*}\right)-I(\theta)$ is the volume between the "surfaces" $J_{k} X_{k}$ and $J_{k}^{*} X_{k}^{*}$ in $T^{2 k+1}$. In the case $k=1$, this variation is computed by the method of integral calculus [BH1, Theorem 5.8]:

Proposition 4.2. Let $\theta_{1}, \theta_{2}$, and $\theta_{3}$ be integral harmonic 1-forms subject to the conditions that $\int_{X} \theta_{i} \wedge \theta_{j}=0$ for all $i, j$. This condition is equivalent to the three form $\theta=\theta_{1} \wedge \theta_{2} \wedge \theta_{3}$ being primitive on the Jacobian $J$ of $X$. For $1 \leq i, j \leq 3$ with $i \neq j$, let $\eta_{i, j}$ be the unique 1-form on $X$ such that $d \eta_{i, j}=\theta_{i} \wedge \theta_{j}$ and $\eta_{i, j}$ is orthogonal to all closed 1-forms on $X$. Define $Q: P \otimes_{\mathbf{Z}} \mathbf{R} \rightarrow H^{0}\left(X ; K^{2}\right)$ by

$$
Q\left(\theta_{1} \wedge \theta_{2} \wedge \theta_{3}\right)=\sum_{(1,2,3)}\left(\theta_{1}+i^{*} \theta_{1}\right)\left(\eta_{2,3}+i^{*} \eta_{2,3}\right)
$$


where $*$ indicates the Hodge star operator and the sum is over cycle permutations of the set $\{1,2,3\}$. Then

Consider the harmonic volume $I(\theta ; s)$ for $s=\left(s_{1}, \ldots, s_{3 g-3}\right)$ near $s=0$.

$$
I(\theta ; s)-I(\theta ; 0)=\operatorname{Im}\left[2 \pi \sum_{j=1}^{3 g-3} s_{j} \frac{Q(\theta)}{\left(d z_{j}\right)^{2}}\left(t_{j}\right)\right]+o(s),
$$

where $z_{j}$ is the complex coordinate on $X=X_{0}$ near $t_{j}$. Further, harmonic volume varies holomorphically with $s$.

We will use this result for the case $k=1$ to derive an analogous formula for the variation of harmonic volume for arbitrary $k$. Our result, which completes this section, is

Theorem 4.3. Define $Q_{1}: G_{\mathbf{R}} \rightarrow H^{0}\left(X ; K^{2}\right)$ by

$$
\begin{aligned}
Q_{1}\left(\theta_{1}\right. & \left.\wedge \cdots \wedge \theta_{2 k+1}\right) \\
= & \sum_{\mu \in S_{2 k+1}}(\operatorname{sgn} \mu)\left(\theta_{\mu(2 k+1)}+i^{*} \theta_{\mu(2 k+1)}\right) \\
& \cdot\left(\eta_{\mu(2 k-1), \mu(2 k)}+i^{*} \eta_{\mu(2 k-1), \mu(2 k)}\right) \prod_{l=1}^{k-1} \int_{X} \theta_{\mu(2 l-1)} \wedge \theta_{\mu(2 l)},
\end{aligned}
$$

where $\eta_{i, j}$ is the unique 1-form on $X$ such that $d \eta_{i, j}=\theta_{i} \wedge \theta_{j}$ and $\eta_{i, j}$ is orthogonal to all closed 1-forms on $X$.

For $s=\left(s_{1}, \ldots, s_{3 g-3}\right) \in \mathbf{C}^{3 g-3}$ of sufficiently small modulus,

$$
I(\theta ; s)-I(\theta ; 0)=\frac{1}{2^{k}} \frac{1}{(k-1) !} \operatorname{Im}\left[2 \pi \sum_{j=1}^{3 g-3} s_{j} \frac{Q_{1}(\theta)}{\left(d z_{j}\right)^{2}}\left(t_{j}\right)\right]+o(s),
$$

where $z_{j}$ is a local coordinate on $X=X_{0}$ around $t_{j}$.

Proof. From the computation in the proof of Lemma 3.3, we have

$$
k ! \nu(\theta)=k \sum_{I \in \mathscr{I}} \varepsilon_{I} \int_{D_{3}} \theta_{i_{1}} \wedge \theta_{i_{2}} \wedge \theta_{i_{3}} \cdot \prod_{l=1}^{k-1} \int_{X} \theta_{i_{2 l+2}} \wedge \theta_{i_{2 l+3}},
$$

where $\mathscr{I}$ is the collection of all multi-indices $I=\left\{i_{1}, \ldots, i_{2 k+1}\right\}$ with $1 \leq$ $i_{1}<i_{2}<i_{3} \leq 2 k+1 ; 1 \leq i_{2 l}<i_{2 l+1} \leq 2 k+1$, for $2 \leq l \leq k$; and $\varepsilon_{I}$ is the sign of the permutation $i_{1}, \ldots, i_{2 k+1}$ of $1, \ldots, 2 k+1$.

Since $\theta$ is $k$-good, we may assume $\theta$ is $k$-orthogonal by Proposition 2.9. Hence, some $k+2$ of the $\theta_{i}$ 's are mutually orthogonal under the skew symmetric pairing $(\alpha, \beta) \mapsto \int_{X} \alpha \wedge \beta$. We may assume that $\theta_{i_{1}}, \theta_{i_{2}}$, and $\theta_{i_{3}}$ are mutually orthogonal, for otherwise the summand is zero. So, $\theta_{i_{1}} \wedge \theta_{i_{2}} \wedge \theta_{i_{3}}$ is a primitive 3-form on $J$. Then

$$
\int_{D_{3}} \theta_{i_{1}} \wedge \theta_{i_{2}} \wedge \theta_{i_{3}}
$$

is simply $\nu(\theta)$, where this $\nu$ is the Abel-Jacobi map on integral 3-forms. 
Now consider $X$ as varying in Torelli space. Then

$$
\begin{aligned}
k !\left[\nu\left(\theta^{*}\right)-\nu(\theta)\right]= & k \sum_{I \in \mathcal{I}} \varepsilon_{I} \int_{D_{3}} \theta_{i_{1}}^{*} \wedge \theta_{i_{2}}^{*} \wedge \theta_{i_{3}}^{*} \cdot \prod_{l=1}^{k-1} \int_{X} \theta_{i_{2 l+2}}^{*} \wedge \theta_{i_{2 l+3}}^{*} \\
& -k \sum_{I \in \mathcal{I}} \varepsilon_{I} \int_{D_{3}} \theta_{i_{1}} \wedge \theta_{i_{2}} \wedge \theta_{i_{3}} \cdot \prod_{l=1}^{k-1} \int_{X} \theta_{i_{2 l+2}} \wedge \theta_{i_{2 l+3}} .
\end{aligned}
$$

Since the pairing $(\alpha, \beta) \mapsto \int_{X} \alpha \wedge \beta$ is topological, and therefore independent of complex structure, the integrals appearing in the products are constant with respect to variation in Torelli space. Hence, we obtain

$$
\begin{aligned}
& k !\left[\nu\left(\theta^{*}\right)-\nu(\theta)\right] \\
& \quad=k \sum_{I \in \mathcal{F}} \varepsilon_{I}\left(\int_{D_{3}} \theta_{i_{1}}^{*} \wedge \theta_{i_{2}}^{*} \wedge \theta_{i_{3}}^{*}-\int_{D_{3}} \theta_{i_{1}} \wedge \theta_{i_{2}} \wedge \theta_{i_{3}}\right) \prod_{l=1}^{k-1} \int_{X} \theta_{i_{2 l+2}} \wedge \theta_{i_{2 l+3}} \\
& \quad=k \sum_{I \in \mathscr{I}} \varepsilon_{I}\left\{\nu\left(\theta_{i_{1}}^{*} \wedge \theta_{i_{2}}^{*} \wedge \theta_{i_{3}}^{*}\right)-\nu\left(\theta_{i_{1}} \wedge \theta_{i_{2}} \wedge \theta_{i_{3}}\right)\right\} \prod_{l=1}^{k-1} \int_{X} \theta_{i_{2 l+2}} \wedge \theta_{i_{2 l+3}},
\end{aligned}
$$

where the $\nu$ 's in the braces signify the Abel-Jacobi map on primitive 3-forms.

Quoting Proposition 4.2 and using the fact that $\nu=2 I$ on good forms, we have

$$
\nu\left(\theta^{*}\right)-\nu(\theta)=2 \operatorname{Im}\left[2 \pi \sum_{j=1}^{3 g-3} s_{j} \frac{Q(\theta)}{\left(d z_{j}\right)^{2}}\left(t_{j}\right)\right]+o(s)
$$

where

$$
Q\left(\theta_{1} \wedge \theta_{2} \wedge \theta_{3}\right)=\sum_{(1,2,3)}\left(\theta_{1}+i^{*} \theta_{1}\right)\left(\eta_{2,3}+i^{*} \eta_{2,3}\right)
$$

and $z_{j}$ is the complex coordinate on $X=X_{0}$ near $t_{j}$. Substituting this into $(*)$, we obtain

$$
\begin{array}{r}
k !\left[\nu\left(\theta^{*}\right)-\nu(\theta)\right] \\
=2 k \sum_{I \in \mathcal{F}} \varepsilon_{I} \operatorname{Im}\left[2 \pi \sum_{j=1}^{3 g-3} s_{j} \frac{\sum_{\left(i_{1}, i_{2}, i_{3}\right)}\left(\theta_{i_{1}}+i^{*} \theta_{i_{1}}\right)\left(\eta_{i_{2}, i_{3}}+i^{*} \eta_{i_{2}, i_{3}}\right)}{\left(d z_{j}\right)^{2}}\left(t_{j}\right)\right] \\
\cdot \prod_{l=1}^{k-1} \int_{X} \theta_{i_{2 l+2}} \wedge \theta_{i_{2 l+3}}+o(s) .
\end{array}
$$

The use of a combinatorial argument and the fact that $\nu$ is twice the value of harmonic volume on good forms now concludes the proof.

The fact that the map $Q_{1}$ has values in the holomorphic quadratic differentials follows from Proposition 4.2.

\section{NONDEGENERACY OF HARMONIC VOLUME}

We first show that the homomorphism $Q_{1}$ of the last section may be interpreted as the codifferential in the direction of the fiber of the mapping $\nu$, considered as a map from Torelli space into the torus bundle with fiber $G_{+}^{*} / G^{*}$. 
Using this it is possible to perform a computation which shows that, for a hyperelliptic Riemann surface $X$ which has its branch points located radially about the origin and symmetrically about the real axis, this codifferential of harmonic volume maps surjectively onto the (-1)-eigenspace of the hyperelliptic involution on the hyperelliptic locus in Torelli space. Thus, the differential of the harmonic volume map at the point of Torelli space represented by $X$ is injective on the subspace of the tangent space to Torelli space which is dual to the $(-1)$-eigenspace of the hyperelliptic involution on the hyperelliptic locus. In particular, whereas harmonic volume is identically zero on primitive forms, it is nondegenerate on good forms. This implies that an intermediate Jacobian based on good forms might be valuable in determining the algebraic equivalence of algebraic cycles.

Let $X$ be a hyperelliptic Riemann surface of genus $g \geq 2 k+1$. Without loss of generality we may assume that $X$ has equation $y^{2}=\prod_{i=1}^{n}\left(x-e_{i}\right)$, where $n=2 g+2$. Let $\omega_{j}=x^{j} d x / y, 0 \leq j \leq g-1$, be a basis for $H^{0}(X ; K)$, where $K$ is the canonical bundle on $X$. Let $x=r e^{i \theta}$ and $x-e_{j}=r_{j} e^{i \theta_{j}}$. We assume $\prod_{j=1}^{n} r_{j}$ is an even function of $\theta$ which is also invariant under the transformation taking $\theta$ to $\theta+2 \pi / n$. This condition is fulfilled if the branch points of $X$ are located radially about the origin with one branch point located on the positive real axis.

A computation [BH1, §6] shows that $\operatorname{Re}\left(\omega_{l}\right) \wedge \operatorname{Re}\left(\omega_{m}\right)$ is a well-defined form on $\mathbf{P}^{1}$ and that integration of this form over $\mathbf{P}^{1}$ yields zero, so we conclude that

$$
\operatorname{Re}\left(\omega_{l}\right) \cdot \operatorname{Re}\left(\omega_{m}\right)=0 \text { for all } l, m .
$$

Similarly, it is shown that

$$
\operatorname{Im}\left(\omega_{l}\right) \cdot \operatorname{Im}\left(\omega_{m}\right)=0 \text { for all } l, m .
$$

We next compute

$$
\begin{aligned}
\operatorname{Re}\left(\omega_{l}\right) \wedge \operatorname{Im}\left(\omega_{m}\right) & =\frac{\omega_{l}+\bar{\omega}_{l}}{2} \wedge \frac{\omega_{m}-\bar{\omega}_{m}}{2 i} \\
& =-\frac{1}{4 i}\left(\omega_{m} \wedge \bar{\omega}_{l}+\omega_{l} \wedge \bar{\omega}_{m}\right) \\
& =-\frac{1}{4 i} \frac{r^{l+m}\left(e^{i(m-l) \theta}+e^{i(l-m) \theta}\right)}{\prod r_{j}}-2 i r d r d \theta \\
& =\frac{r^{l+m} \cos (m-l) \theta}{\prod r_{j}} r d r d \theta,
\end{aligned}
$$

which is also a well-defined form on $\mathbf{P}^{1}$. Thus, we have

$$
\int_{X} \operatorname{Re}\left(\omega_{l}\right) \wedge \operatorname{Im}\left(\omega_{m}\right)=2 \int_{\theta=0}^{2 \pi} \int_{r=0}^{\infty} \frac{r^{l+m} \cos (m-l) \theta}{\prod r_{j}} r d r d \theta .
$$

Since the function $\prod r_{j}$ is presumed to be an even function of $\theta$ which is also invariant under the transformation taking $\theta$ to $\theta+2 \pi / n$, we conclude that it is also invariant under the transformation taking $\theta$ to $\theta-2 \pi / n$. Now 
we compute

$$
\begin{aligned}
& \int_{\theta=0}^{2 \pi} \frac{r^{l+m}}{\prod r_{j}} \cos [(m-l) \theta] d \theta=\int_{\theta=2 \pi / n}^{2 \pi+2 \pi / n} \frac{r^{l+m}}{\prod r_{j}} \cos \left[(m-l)\left(\theta-\frac{2 \pi}{n}\right)\right] d \theta \\
&=\int_{2 \pi / n}^{2 \pi+2 \pi / n} \frac{r^{l+m}}{\prod r_{j}}\left\{\cos [(m-l) \theta] \cos \left[(m-l) \frac{2 \pi}{n}\right]\right. \\
&\left.\quad+\sin [(m-l) \theta] \sin \left[(m-l) \frac{2 \pi}{n}\right]\right\} d \theta \\
&=\cos \left[(m-l) \frac{2 \pi}{n}\right] \int_{2 \pi / n}^{2 \pi+2 \pi / n} \frac{r^{l+m}}{\prod r_{j}} \cos [(m-l) \theta] d \theta \\
&=\cos \left[(m-l) \frac{2 \pi}{n}\right] \int_{\theta=0}^{2 \pi} \frac{r^{l+m}}{\prod r_{j}} \cos \left[(m-l)\left(\theta+\frac{2 \pi}{n}\right)\right] d \theta \\
&=\cos \left[(m-l) \frac{2 \pi}{n}\right] \int_{\theta=0}^{2 \pi} \frac{r^{l+m}}{\prod r_{j}} \cos [(m-l) \theta] d \theta .
\end{aligned}
$$

We conclude that if $\operatorname{Re}\left(\omega_{l}\right) \cdot \operatorname{Im}\left(\omega_{m}\right) \neq 0$, then $(m-l) 2 \pi / n$ is an integral multiple of $\pi$, or equivalently, $m-l$ is an integral multiple of $g+1$. Since $0 \leq m, l \leq g-1$, this forces $m=l$. So, we obtain

$$
\operatorname{Re}\left(\omega_{l}\right) \cdot \operatorname{Im}\left(\omega_{m}\right)=0 \text { for } l \neq m \text {. }
$$

Any holomorphic 1-form $\omega$ can be written $\omega=\theta+i^{*} \theta$ where $\theta$ is a harmonic 1-form. Further, since $* *=-1$, it is easily seen that ${ }^{*} \omega=-i \omega$. The Hodge star operator defines a hermitian positive definite bilinear form on $H^{1,0}(X)$ given by

$$
\langle\phi, \psi\rangle=\int_{X} \phi \wedge^{*} \bar{\psi}=i \int_{X} \phi \wedge \bar{\psi}
$$

This is called the Hodge inner product. It is easily computed that

$$
\langle\phi, \psi\rangle=0 \text { if and only if } \operatorname{Re}(\phi) \cdot \operatorname{Re}(\psi)=\operatorname{Re}(\phi) \cdot \operatorname{Im}(\psi)=0 .
$$

Fix $j$ with $0 \leq j \leq g-3$. Let $\omega_{1}^{\prime}=\omega_{j}, \omega_{2}^{\prime}=\omega_{j+1}$, and $\omega_{3}^{\prime}=$ $\omega_{j+2}$. It follows from the preceding paragraph and our preceding computations that $\omega_{1}^{\prime}, \omega_{2}^{\prime}$, and $\omega_{3}^{\prime}$ are mutually orthogonal holomorphic 1-forms under the Hodge inner product. We now complete $\omega_{1}^{\prime}, \omega_{2}^{\prime}, \omega_{3}^{\prime}$ to a basis $\omega_{1}^{\prime}, \ldots, \omega_{g}^{\prime}$ for $H^{1,0}(X)$ consisting of pairwise orthogonal holomorphic 1forms. For $1 \leq i \leq 3$, let $\theta_{i}=\operatorname{Re}\left(\omega_{i}^{\prime}\right)$. Further, let $\theta_{2(i-2)}=\operatorname{Re}\left(\omega_{i}^{\prime}\right)$ and $\theta_{2(i-2)+1}=\operatorname{Im}\left(\omega_{i}^{\prime}\right)$ for $4 \leq i \leq k+2$. Since $\omega_{1}^{\prime}, \ldots, \omega_{g}^{\prime}$ are pairwise orthogonal under the Hodge inner product, we have $\operatorname{Re}\left(\omega_{l}^{\prime}\right) \cdot \operatorname{Re}\left(\omega_{m}^{\prime}\right)=0$ for all $l, m$ and $\operatorname{Re}\left(\omega_{l}^{\prime}\right) \cdot \operatorname{Im}\left(\omega_{m}^{\prime}\right)=0$ for all $l \neq m$. It follows that

$$
\theta_{i} \cdot \theta_{j}=0
$$

if $\{i, j\} \neq\{2 m+2,2 m+3\}$ for some $1 \leq m \leq k-1$. It also follows that $\theta_{1}, \theta_{2}, \theta_{3}$, and $\theta_{2 l}$, for $2 \leq l \leq k$, are $k+2$ mutually orthogonal 1-forms, so that $\theta_{1} \wedge \cdots \wedge \theta_{2 k+1}$ is good, by Proposition 2.9.

Recall the formula in Theorem 4.3 computing the change in harmonic volume, a formula which we now denote by $\delta I$. Since $\nu=2 I$, we may interpret this map as the "vertical codifferential" of the map $\nu$, considered as a section of the holomorphic torus bundle with fiber $G_{+}(s)^{*} / G(s)^{*}$ as $s$ varies in a 
disk $\Delta$ in Torelli space. In order to see this interpretation, recall that the fiber of this bundle is actually the real dual space of $G_{\mathbf{R}}$, and we have endowed $G_{\mathbf{R}}$ with a complex structure making it canonically complex-isomorphic to $F^{k+1} G_{\mathbf{C}}$. Hence, over a small $(3 g-3)$-dimensional disk in Torelli space, the torus bundle is analytically trivial, and we may identify all the fibers over $\Delta$ with a single fiber, say over a point $p$ in Torelli space representing a Riemann surface $X$, and then reintroduce the complex structure on that fiber via the identification of $G_{\mathbf{R}}$ with $G_{+}(p)^{*} / G(p)^{*}$. (See [G2, §II.1].) Thus, we may consider $\nu$ as mapping $\Delta$ into a fixed fiber, and then $\delta I$ (modulo a factor of two) represents the codifferential

$$
G_{+} \rightarrow T_{p}^{*}(\Delta) \cong H^{0}\left(X, \Omega^{2}\right)
$$

By Theorem 4.3,

$$
\begin{aligned}
\delta I= & \sum_{\mu \in S_{2 k+1}}(\operatorname{sgn} \mu)\left(\theta_{\mu(2 k+1)}+i^{*} \theta_{\mu(2 k+1)}\right) \\
& \cdot\left(\eta_{\mu(2 k-1), \mu(2 k)}+i^{*} \eta_{\mu(2 k-1), \mu(2 k)}\right) \prod_{l=1}^{k-1}\left(\theta_{\mu(2 l-1)} \cdot \theta_{\mu(2 l)}\right)
\end{aligned}
$$

and, using what we know about $\left(\theta_{i} \cdot \theta_{j}\right)$ from equation $(*)$, we obtain

$$
\begin{aligned}
\delta I= & 2^{k-1}(k-1) ! \sum_{\tau \in S_{3}}(\operatorname{sgn} \tau)\left(\theta_{\tau(3)}+i^{*} \theta_{\tau(3)}\right) \\
& \cdot\left(\eta_{\tau(1), \tau(2)}+i^{*} \eta_{\tau(1), \tau(2)}\right) \prod_{l=1}^{k-1}\left(\theta_{2 l+2} \cdot \theta_{2 l+3}\right) .
\end{aligned}
$$

The computation done in $\S 5$ of [BH1] now allows us to conclude that $\delta I$ maps indecomposable good forms onto the space of quadratic differentials of the form $x^{j}(d x)^{2} / y$, where $0 \leq j \leq g-3$.

This set of holomorphic quadratic differentials forms a basis for the $(-1)$ eigenspace of the hyperelliptic involution on the hyperelliptic locus in Torelli space. It follows that the differential of the mapping $\nu$ is injective on the dual space of this $(-1)$-eigenspace. In particular, the section $\nu$ is not identically zero, and therefore generically nonzero by analyticity.

We remark that a careful examination of the proof of this result shows that we actually need only take $g \geq k+2$ and that the real $(2 k+1)$-forms used in the computation correspond to $(k+2, k-1)$-forms in $G_{+}$, on which $\nu$ must vanish if $W_{k}$ is algebraically equivalent to $W_{k}^{-}$. Presumably with additional knowledge on the value of the change in harmonic volume on $(k+1, k)$-forms lying in the image of the differentiation of $\nu$ with respect to a generic deformation of $X$ in Torelli space, an independent proof of Ceresa's theorem might be achieved.

\section{REFERENCES}

[BH1] B. Harris, Harmonic volumes, Acta Math. 150 (1983), 91-123.

[BH2] - Homological versus algebraic equivalence in a Jacobian, Proc. Nat. Acad. Sci. U.S.A. 80 (1983), 1157-1158.

[BH3] _ A triple product for automorphic forms, Quart. J. Math. Oxford 34 (1983), 67-75. 
[C] G. Ceresa, $C$ is not algebraically equivalent to $C^{-}$in its Jacobian, Ann. of Math. (2) 117 (1983), 285-291.

[CC] G. Ceresa and A. Collino, Some remarks on algebraic equivalence of cycles, Pacific J. Math. 105 (1983), 285-290.

[Co] A. Collino, Poincaré's formulas and hyperelliptic curves, Atti Torino 109 (1975), 89-101.

[G1] P. Griffiths, Periods of integrals on algebraic manifolds. I, Amer. J. Math. 90 (1968), 568626.

[G2] —-, Periods of integrals on algebraic manifolds. II, Amer. J. Math. 90 (1968), 805-865.

[G3] _- On the period of certain rational integrals. I, Ann. of Math. (2) 90 (1969), 460-495.

[G4] _ On the period of certain rational integrals. II, Ann. of Math. 90 (1969), 496-541.

[K] K. Kodaira, Complex manifolds and deformation of complex structure, Springer-Verlag, New York, 1986.

[M] I. G. MacDonald, Symmetric products of an algebraic curve, Topology 1 (1962), 319-343.

[P] M. Pulte, The fundamental group of a Riemann surface: Mixed Hodge structures and algebraic cycles, Duke Math. J. 57 (1988), 721-760.

[SS] M. Schiffer and D. C. Spencer, Functionals of finite Riemann surfaces, Princeton Univ. Press, Princeton, N.J., 1954.

[W] A. Weil, Collected papers, Oeuvres Scientifique 2, 533-534.

Department of Mathematics, Northeast Missouri State University, Kirksville, MisSOURI 63501

E-mail address: mt30@nemomus.bitnet 